\title{
Mapping quasifission characteristics and timescales in heavy element formation reactions
}

\author{
R. du Rietz, ${ }^{*}$ E. Williams, D. J. Hinde, ${ }^{\dagger}$ M. Dasgupta, M. Evers, C. J. Lin, ${ }^{\dagger}$ D. H. Luong, C. Simenel, and A. Wakhle \\ Department of Nuclear Physics, Research School of Physics and Engineering, The Australian National University, ACT 0200, Australia
}

(Received 12 September 2013; published 25 November 2013)

\begin{abstract}
Background: The formation of superheavy elements by fusion of two massive nuclei is severely inhibited by the competing quasifission process.

Purpose: Through extensive mass-angle distribution measurements, we map out the systematic dependence of quasifission characteristics as a function of the identity of the colliding nuclei.

Methods: The Australian National University's Heavy Ion Accelerator Facility and CUBE spectrometer have been used to measure mass-angle distributions for 42 reactions forming heavy elements. Beam energies above their respective capture barriers were used to minimize the known influence of nuclear structure effects.

Results: Different mappings of mass-angle distribution characteristics (including timescales) over the nuclear landscape show a systematic dependence on entrance channel and compound nucleus fissilities.

Conclusions: The results provide an empirical baseline to assess effects of nuclear structure at lower beam energies, and motivate the testing and validation of complete dynamical models of heavy element fusion through comparison of mass-angle distributions.
\end{abstract}

DOI: 10.1103/PhysRevC.88.054618

PACS number(s): 25.70.Jj, 25.70.Gh

\section{INTRODUCTION}

The formation of heavy and superheavy elements provides access to extreme nuclear systems in which our understanding of the quantum many-body physics governing nuclear matter can be tested. Research into their formation has provided important insights into the dynamics of the nuclear fusion process. However, our ability to plan the production of such nuclei-either in greater quantity or in as yet unexplored regions of the nuclear chart-is hindered by a significant gap in our understanding of the mechanism of heavy element formation. This gap lies in the dynamical evolution that the system undergoes after contact of the two heavy colliding nuclei, as discussed below.

Heavy element formation can be divided into three steps. Each step influences formation cross sections, and is therefore important in defining our ability to produce such nuclei. First, two colliding nuclei must have enough kinetic energy to overcome the repulsive Coulomb barrier, and approach close enough for the short-range attractive nuclear force to come into play. This allows capture inside the barrier. Next, the newly formed dinuclear system must undergo shape evolution towards a compact mononuclear shape near the equilibrium deformation. Finally, the newly formed compound system must survive statistical (compound nucleus) fission to form an evaporation residue.

The cross section for heavy element formation via fusionevaporation is given by

$$
\sigma_{\mathrm{ER}}=\sum_{J=0}^{\infty} \sigma_{J}\left(E_{\mathrm{c} . \mathrm{m} .}, J\right) P_{\mathrm{CN}}\left(E^{*}, J\right) W_{\mathrm{sur}}\left(E^{*}, J\right),
$$

\footnotetext{
*Current address: Malmö University, Faculty of Technology and Society, 20506 Malmö, Sweden.

†Corresponding author: david.hinde@anu.edu.au

${ }^{\ddagger}$ Current address: China Institute of Atomic Energy, P.O. Box 275(10), Beijing 102413, China.
}

where $\sigma_{J}\left(E_{\mathrm{c} . \mathrm{m} .}, J\right)$ is the capture cross section as a function of center-of-mass energy $E_{\text {c.m. }}$ and angular momentum $J \hbar$, $P_{\mathrm{CN}}\left(E^{*}, J\right)$ is the probability that the system reaches the equilibrium configuration as a function of the excitation energy $E^{*}$ and $J$, and $W_{\text {sur }}\left(E^{*}, J\right)$ is the probability that the system survives statistical fission decay through sequential particle evaporation, thus eventually forming the desired (super)heavy evaporation residue.

There are two potential outcomes after capture: fusion, in which the dinuclear system evolves in shape to form a compact equilibrated heavy nucleus, and quasifission, the rapid fission-like decay of the elongated system before a compact compound nucleus can be formed [1-3]. The competition between these two outcomes exhibits complex behavior. It has been shown to be heavily influenced by entrance channel properties such as the charge product (or mass asymmetry) in the entrance channel [4-7], deformation alignment [8-12], spherical closed shells, and the asymmetry of the projectile and target $N / Z$ ratios [13]. All these dependencies give insights into the surprisingly intricate physical process of shape evolution, which results either in formation of a compact compound nucleus or in quasifission.

Capture and statistical fission decay, the processes represented by the first and third terms in Eq. (1), can in principle be described using relatively simple concepts and theoretical expressions. Energy balance and statistical decay are the central concepts used to model the physics of these processes, respectively, and are used to calculate these terms in the equation $[14,15]$.

The probability $P_{\mathrm{CN}}\left(E^{*}, J\right)$ that the system reaches a compact equilibrium shape, thus forming a compound nucleus [the second term of Eq. (1)], is considered to be both the most complex [16] and least understood factor determining heavy element formation cross sections. It is also the most important factor in Eq. (1), in two senses. The first is that quasifission (sometimes called nonequilibrium fission) is generally the dominant outcome in superheavy element formation reactions 
(i.e., $\left.P_{\mathrm{CN}} \ll 1\right)$. The second is that many different beam-target combinations, with differing $P_{\mathrm{CN}}\left(E^{*}, J\right)$, can in principle be used to form the same compound nucleus, whereas the fission survival probability is characteristic of the nucleus to be formed, and cannot be changed (except through the excitation energy).

\section{A. Addressing a complex problem}

Determination of $P_{\mathrm{CN}}$ is a difficult challenge for two reasons. The first is that unambiguous experimental information on $P_{\mathrm{CN}}$ is hard to obtain (though it is possible in favorable cases [6]). The second is that theoretical calculations of $P_{\mathrm{CN}}$ values should take into account all of the many entrancechannel variables now implicated [13] in determining $P_{\mathrm{CN}}$. Both problems must be addressed in order to maximize our ability to create and study superheavy elements.

\section{Experimental problems}

Experimental values of $P_{\mathrm{CN}}$ cannot be determined directly from heavy element cross sections, since the latter is also strongly dependent on the last term in Eq. (1), describing statistical fission competition. Despite its conceptual simplicity, probabilities $W_{\text {sur }}\left(E^{*}, J\right)$ depend exponentially on key parameters, which are not always well known. It has been shown [17] that there can be orders of magnitude variation in values of $W_{\text {sur }}$ calculated for the same system. This uncertainly then carries over into extracted values of $P_{\mathrm{CN}}$. This means values of $P_{\mathrm{CN}}$ cannot reliably be determined experimentally from $\sigma_{\mathrm{ER}}$ unless the value of $W_{\text {sur }}$ is either constrained or can be canceled by analyzing yields from several reactions forming the same compound nucleus [5-7].

\section{Theoretical problems}

Theoretical calculation of $P_{\mathrm{CN}}$ is challenging because of the complex nonequilibrium dynamics involved in the collective motion from contact to compact configurations. This makes a priori predictions of $P_{\mathrm{CN}}$ from theoretical models difficult. A sophisticated theoretical approach to treat this problem is by modeling diffusion over a complicated multidimensional potential energy surface (PES) [18-20]. Other approaches have resulted in simpler models [21] for $P_{\mathrm{CN}}$, some using energetics and statistical concepts [22]. Recently the fully quantum microscopic TDHF approach [13] has been applied to heavy element formation reactions. No model approach is currently predominant. Those that perform better for some cases are often limited in the type of reactions and/or observables they can predict. Those that offer a more generally applicable approach require vast parameter spaces, and may not yet include a dynamical treatment of all important aspects of the physics [23].

\section{The ultimate goal}

What is needed is a model that can reliably predict $P_{\mathrm{CN}}$ for any reaction. This is a major task, and significant developments are taking place in this direction $[16,23]$. However, efforts to test models through comparison of calculated and experimental $P_{\mathrm{CN}}$ values are complicated by the interplay between capture, quasifission, and statistical fission, as demonstrated in Eq. (1). To guarantee that models correctly include the physics relevant to heavy element formation, they should be validated by comparing their predictions of the characteristics of the complementary quasifission process against observations. In particular, reproducing observable quantities that are sensitive to reaction timescales should be a critical challenge of a nonequilibrium model.

\section{B. Mapping quasifission timescales}

The observable giving the most direct information on quasifission timescales is the experimental mass-angle distribution (MAD). This shows the mass distribution of fission fragments as a function of the center-of-mass scattering angle $\theta_{\text {c.m. }}$. In this work we define the MAD as the distribution $d^{2} \sigma / d \theta_{\text {c.m. }} d M_{R}$, where $\sigma$ is the cross section and $M_{R}$ is the binary fission mass ratio. Mass-angle distributions [2,24] reveal quasifission timescales in the important time range of $10^{-21} \mathrm{~s}$ to $10^{-20} \mathrm{~s}$ in the most model-independent way.

With the recent extensive program of measurements of mass-angle distributions at the Australian National University, there is now the opportunity to take a new approach to investigating heavy element fusion dynamics-one that concentrates on the systematic behavior of experimental massangle distributions (and thus reaction timescales) over a very broad range of reactions.

The approach begins by choosing measurements at beam energies somewhat above the capture barrier (typically by $\sim 6 \%$ ). Here the known effects of deformation alignment $[8,9,25,26]$ and shell structure observed in measurements at below-barrier energies [13,27] are much reduced [11,25,28]. However the beam energies should not be too far above the capture barriers; otherwise high angular momenta would be introduced in the collisions. This is undesirable for two reasons. The first is empirical: the measurements should still be representative of the energies and angular momenta used in heavy element production reactions. The second is that for comparison with models aiming to predict $P_{\mathrm{CN}}$, the mass-angle distributions should largely reflect the competition between the Coulomb and nuclear potentials, rather than being dominated by the repulsive angular-momentum-dependent centrifugal potential.

The current data set should therefore provide an ideal first test of models of reaction dynamics. Only after reproducing the observed broad trends found in these mass-angle distributions should model calculations be compared to measurements taken at lower beam energies, where deviations from these trends due to nuclear structure effects are expected [13,25,26].

This approach - the identification of broad trends, followed by exploration of finer detail- finds an analogy with the liquid drop model of nuclear masses, in which localized shell effects can be quantified when the underlying smooth trends are well defined.

In this work, we present a systematic study of experimental quasifission mass-angle distributions. Our focus is on how 
quasifission characteristics and timescales evolve as a function of the overall competition between repulsive Coulomb and attractive nuclear forces. This balance changes as the identities of the colliding nuclei are changed. In carrying out this study, we have four goals:

(i) to achieve an improved empirical understanding of the global dependence of quasifission characteristics on the entrance channel;

(ii) to provide experimentally based guidance on which reactions are best suited for producing heavy or superheavy elements;

(iii) by focusing on measurements somewhat above the capture barrier energy, to provide a smooth baseline to quantify the effects of different nuclear structure in the entrance channel, seen at lower bombarding energies; and

(iv) to simplify testing of dynamical models of the competition between quasifission and fusion in heavy element formation.

\section{THE EXPERIMENTS}

The measurements of mass-angle distributions (MAD) were carried out at the Australian National University's Heavy Ion Accelerator Facility, using the 14UD electrostatic tandem accelerator and superconducting linear post-accelerator.

\section{A. Beams and targets}

Beams ranging in mass from ${ }^{12} \mathrm{C}$ to ${ }^{64} \mathrm{Ni}$ bombarded isotopically enriched targets spanning the mass range from ${ }^{144} \mathrm{Sm}$ to ${ }^{238} \mathrm{U}$. Thicknesses ranged from a few tens to a few hundreds of $\mu \mathrm{g} / \mathrm{cm}^{2}$. Generally, the target material was supported by a $\sim 15 \mu \mathrm{g} / \mathrm{cm}^{2}$ carbon (or rarely $\sim 30 \mu \mathrm{g} / \mathrm{cm}^{2}$ aluminium) backing, facing downstream. The target normal was oriented at $60^{\circ}$ to the beam axis for detector configuration $A$, and $45^{\circ}$ for configuration $B$ (see below). This choice of orientation was made in order to minimize the energy loss of fission fragments in the target. Beam energies and projectile-target combinations are listed in Table I. Pulsed beams were used, which had a typical pulse FWHM of $0.7-1.5 \mathrm{~ns}$, and a pulse separation of $107 \mathrm{~ns}$. For the ${ }^{64} \mathrm{Ni}$ beams, the pulse width was considerably greater, and hence the data analysis used the relative time method [29] described in Appendix A.

\section{B. Detectors}

Reaction products were detected using the CUBE spectrometer [9], consisting of two large-area position-sensitive multiwire proportional counters (MWPCs). Each had an illuminated area of $279 \times 357 \mathrm{~mm}^{2}$. A schematic view of the experimental setup is given in Fig. 1.

The MWPCs were placed such that the normal to the central timing foil of each detector was located $180 \mathrm{~mm}$ from the target, at an azimuthal angle $\phi_{1}=180^{\circ}$ for MWPC1 (back) and $\phi_{2}=0^{\circ}$ for MWPC2 (front). The back angle detector was placed in one of three configurations. In the first configuration the detector body was centered at $\theta_{1}=90^{\circ}$. The delay chips could be located either at backward or at forward angles, giving angular coverage of $50^{\circ} \leqslant \theta_{1} \leqslant 125^{\circ}$ or $55^{\circ} \leqslant \theta_{1} \leqslant 130^{\circ}$, respectively. In the second configuration (2) the detector body was centered at $\theta_{1}=135^{\circ}$ giving an angular coverage of $95^{\circ} \leqslant \theta_{1} \leqslant 170^{\circ}$. In all experiments the forward angle detector was placed at a scattering angle $\theta_{2}=45^{\circ}$, providing an angular acceptance of $5^{\circ} \leqslant \theta_{2} \leqslant 80^{\circ}$. Depending on the reaction and the geometry of the backward angle detector, a disk could be placed to mask the forward angle detector at angles close to the beam axis. The maximum angle was chosen to minimize the blocking of coincident fission events.

Position information was obtained from the MWPC detectors using a delay line readout from both ends of the MWPC $X$ and $Y$ position wire planes, which allowed the determination of the position of fission fragments with a resolution of FWHM $\approx 1 \mathrm{~mm}$, which is similar to the beam spot size. Time information, related to each fragment's time-of-flight, was obtained using fast timing signals from the cathode foil of each detector, measured relative to the master RF signal used to generate the pulsed beam. Two silicon surface-barrier detectors (monitors), mounted symmetrically in the vertical plane $\left(\phi \pm 90^{\circ}\right)$ at $\theta=22.5^{\circ}$ or $\theta=30^{\circ}$, recorded elastic scattering to check beam energies and for yield normalization.

The information from the two MWPC detectors was then used to determine the mass ratio of all binary events, assuming two-body kinematics, as described in Appendix A. The mass ratio $M_{R}$ is thus defined in terms of the masses $m_{1}$ and $m_{2}$ of the two fragments as they are repelled in their mutual Coulomb field, through the expression

$$
\begin{aligned}
& M_{R}=\frac{m_{1}}{m_{1}+m_{2}} . \\
& \text { C. Data collection }
\end{aligned}
$$

Data were collected event-by-event, generally triggered by a signal in the back detector, or by a coincidence between front and back. Each trigger started the acquisition of a binary event consisting of positions, energy loss, and time of arrival at each detector. Trigger rates varied from 100 per second to 2000 per second.

\section{MAPPING MASS-ANGLE DISTRIBUTIONS}

Following the methods and procedures described in Appendix A, MADs were extracted for all the reactions listed in Table I. The beam energies of the chosen measurements were typically 5\%-10\% above the capture barrier energy for each reaction, as predicted by the model of Swiatecki [30]. The only exceptions are the reactions with ${ }^{16} \mathrm{O}$, where the data were taken from a short series of measurements on a range of targets at a fixed beam energy $\left(E_{\text {beam }}=111 \mathrm{MeV}\right)$ which resulted in $E / V_{B}$ being $\sim 40 \%$ above the respective barriers. The remaining 36 measurements have a mean $E / V_{B}$ of 1.06 , with standard deviation 0.02 . As pointed out above, the MADs presented show mass ratios rather than masses, since that is what the experimental technique provides (see also Appendix A). 
TABLE I. Reactions for which MAD are presented in this work. The reaction number is referred to in the paper. Capture barrier energies in the center-of-mass frame $V_{\mathrm{b}}$ were calculated using Ref. [30]. Fissilities are calculated for the compound system $\left(x_{\mathrm{CN}}\right)$, and the effective fissility for the target projectile $\left(x_{\text {eff }}\right)$. The classification of the MAD (see Sec. III B) is given in the second last column. Where MAD measurements for this (or a very similar) reaction have been previously described, the reference is given in the last column.

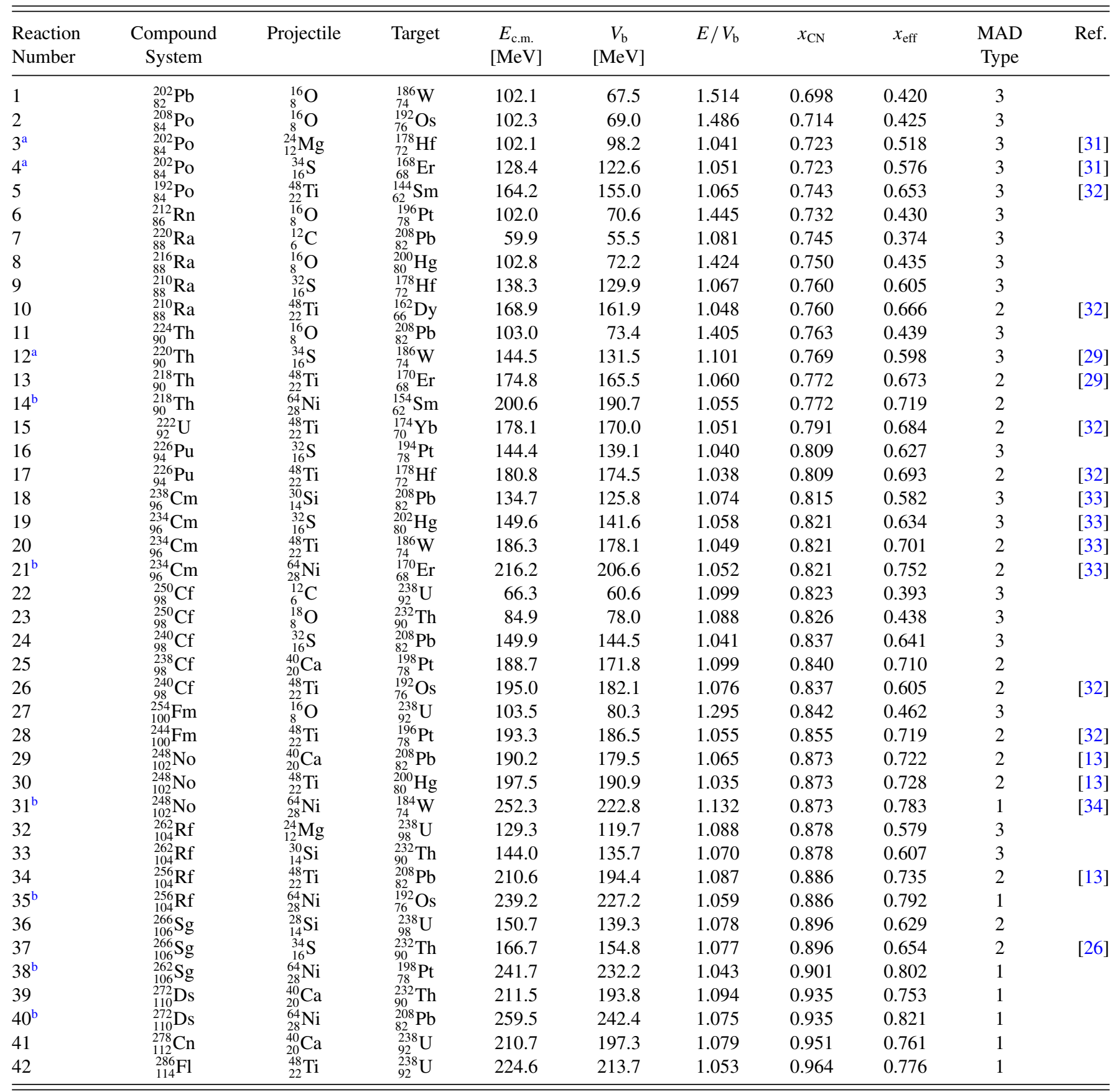

${ }^{\mathrm{a} C U B E}$ detectors in configuration 2.

${ }^{\mathrm{b}}$ Experiment analyzed using relative time method.

As shown in Table I, almost all measurements were carried out using the CUBE MWPC detectors in configuration $A$, and were analyzed using the absolute time method (Appendix A). The few cases using the detectors in configuration $B$ and/or analyzed using the relative time method (Appendix A) are indicated.
To present the systematic changes in MAD across such a wide range of reactions, we have chosen the simple yet informative representation shown in Fig. 2. Here the $x$ axis represents the atomic number of the projectile $\left(Z_{p}\right)$, whose identities are given at the bottom in red. The red dotted lines join reactions with the same $Z_{p}$. The $y$ axis represents 


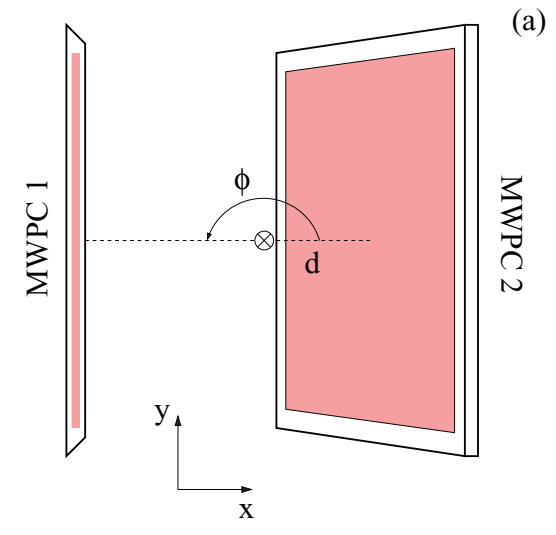

(a)
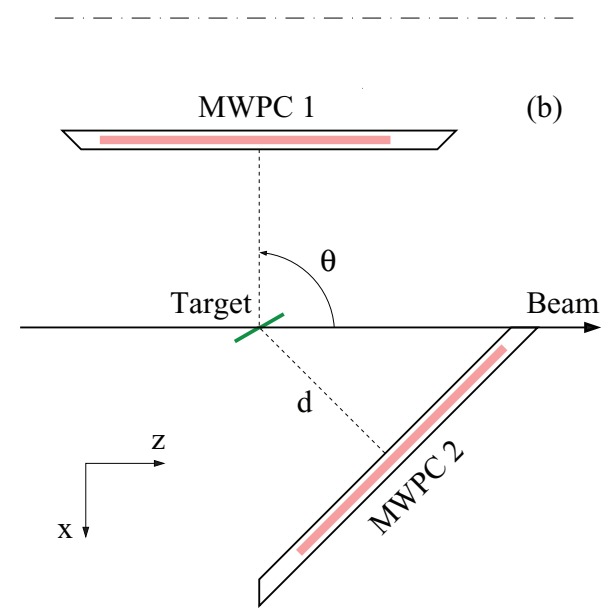

FIG. 1. (Color online) Schematic view of the experimental setup of the CUBE detectors. Panel (a) shows the azimuthal positioning where the beam is going into the page and (b) illustrates the configuration as seen from above. The placement of the detectors is defined by the distance $d$ of their centers to the target, together with their azimuthal angle $(\phi)$, and polar angle $(\theta)$ relative to the beam axis.

the atomic number of the compound nucleus $Z_{\mathrm{CN}}$, whose identities are given in black next to the left-hand axis. Since the bombarding energies are generally less than $10 \%$ above the capture barrier, where pre-equilibrium emission is expected to be negligible, it is safe to assign $Z_{\mathrm{CN}}$ as simply the sum of the projectile and target atomic numbers $Z_{p}+Z_{t}$. The horizontal dotted black lines join reactions forming compound nuclei of the same element, which is identified in black next to the axis on the left. In many cases, reactions forming the same element also formed the same isotope (see Table I).

The locus of reactions with target nuclei having the same atomic number $Z_{t}$ are diagonals in the graph, indicated by the blue dotted lines. The target elements are identified in blue next to the right-hand axis. At the intersection between the three dotted lines, the MAD plot for the corresponding reaction, whose details are given in Table I, is presented.

For clarity, the axis labels have been removed from these smaller MAD plots within the figure; they all correspond in format to the larger MAD example shown in the top left of the figure.

\section{FEATURES OF EXPERIMENTAL MASS-ANGLE DISTRIBUTIONS}

In this section, important aspects of the experimental MADs presented here will be discussed. First, the effects that instrumental properties have on MADs will be reviewed. Next, the reaction mechanisms that contribute to the MAD will be identified, and the physical mechanisms responsible for their differing contributions to each MAD will be described. Finally, three categories of MADs will be defined, in order to simplify the subsequent discussion of the systematic trends observed in the mass-angle distribution characteristics presented in Fig. 2.

\section{A. Instrumental features}

A key feature of the two MWPC detectors used in this study is their wide angular acceptance, allowing a large part of the full angular range of the $\operatorname{MAD}\left(0^{\circ}\right.$ to $\left.180^{\circ}\right)$ to be captured in a single measurement. The scattering angle coverage of the experimental MAD is determined by the size and placement of the two MWPC detectors, each of which covered $75^{\circ}$ in scattering angle. Figure 3 shows the measured MAD for the reaction $E_{\text {beam }}=111 \mathrm{MeV}{ }^{16} \mathrm{O}+{ }^{196} \mathrm{Pt}$. The gray shaded area indicates the region that was not visible due to the binary fragment acceptance of the detectors. As described in detail in Ref. [29], the binary events are simultaneously mirrored about $M_{R}=0.5$ and $\theta_{\text {c.m. }}=90^{\circ}$. The two white points indicate how a single binary event populates the MAD at the complementary mass ratios and center-of-mass scattering angles.

All MADs presented have a constant acceptance in $\phi$, independent of $\theta$, arising either from the placement of the backward angle detector or from gating applied in the analysis. Thus the intensity scale in all the MADs is proportional to $d \sigma / d \theta_{\text {c.m. }}$, rather than $d \sigma / d \Omega_{\text {c.m. }}$. A distribution of $d \sigma / d \Omega_{\text {c.m. }}$. proportional to $1 / \sin \theta_{\text {c.m. }}$. would thus appear uniformly distributed in $\theta_{\text {c.m. }}$. within the MADs.

Inside the angular range covered by the measurements, the major features seen in the MAD plots in Fig. 2 result from a number of different processes. These are elastic and inelastic scattering and transfer reactions (collectively referred to as quasielastic events), deep inelastic reactions, quasifission, and fusion-fission. Ideally all the patterns observed in a given MAD should relate only to the probabilities of these conceptually distinct [35] reaction outcomes. However, there are two instrumental effects that can distort this idealized picture. It is important to note these do not affect the fusion-fission or quasifission components of the MAD.

The first instrumental effect comes through the deliberate rejection of elastically scattered particles where possible. For the reactions with the lighter projectiles ${ }^{12} \mathrm{C}$ and ${ }^{16,18} \mathrm{O}$, essentially the only events visible in the MAD come from fission. For reactions involving these projectiles only, the detector electronic thresholds could be set to exclude the small signals from beam-like particles without excluding any fission events, helped by the small center-of-mass velocity of the fissioning system. This is why no such events are seen in Fig. 3. For all other projectiles, beam-like particles were not rejected, in order that any fission-like events with low pulse height would not also be rejected, thus avoiding distortion 


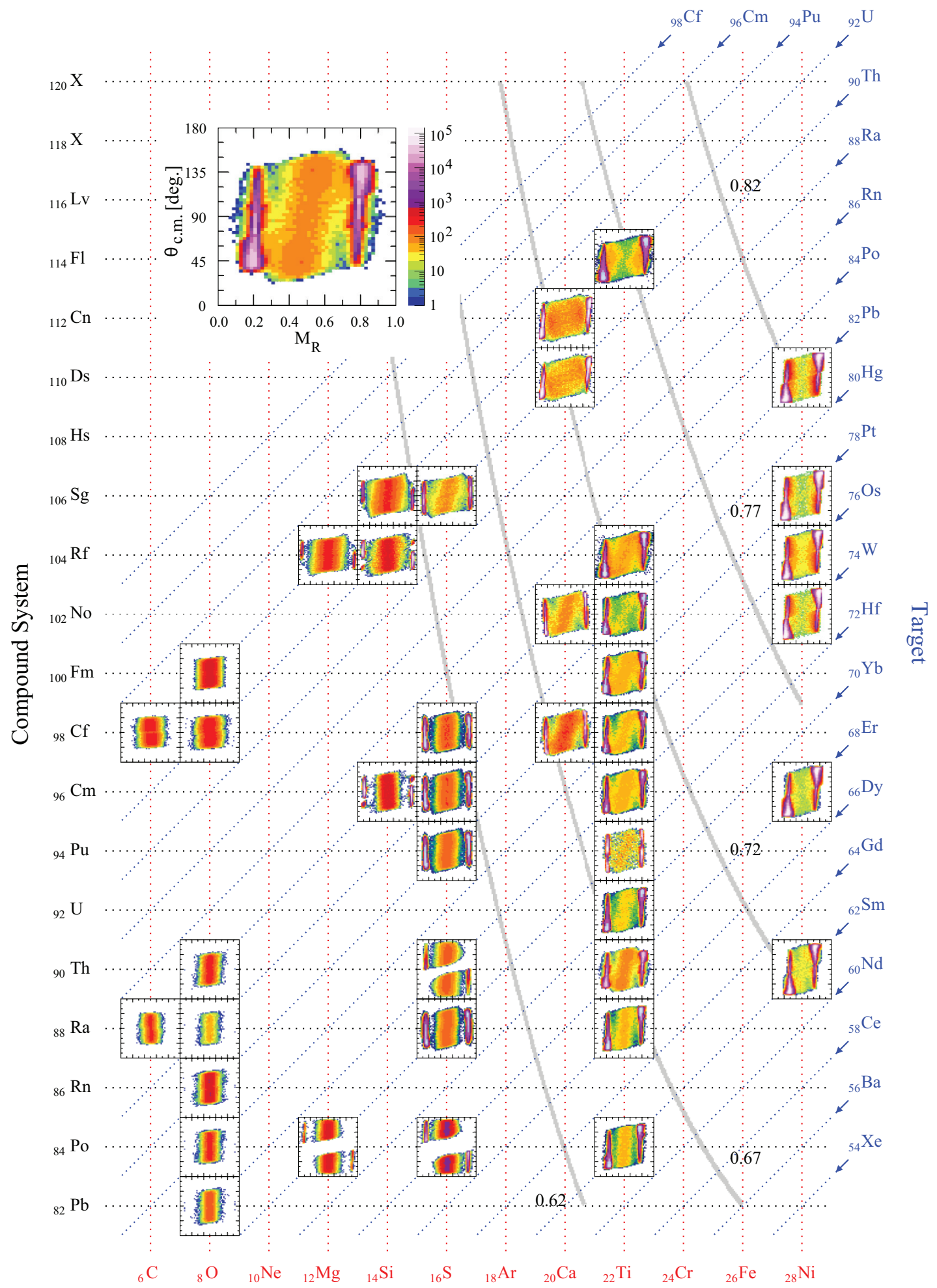

Projectile

FIG. 2. (Color online) MAD scatter plot for all reactions listed in Table I. All miniature plots have the same axes as the large MAD (top left, ${ }^{48} \mathrm{Ti}+{ }^{170} \mathrm{Er}$ at $E_{\text {beam }}=225.0 \mathrm{MeV}$ ). Red vertical and blue diagonal dashed lines correspond to the same projectile and target atomic number, respectively, while the horizontal black dotted lines indicate the compound nucleus atomic number. The full gray lines correspond to the noted constant values of $x_{\text {eff }}$, the entrance channel effective fissility (see text). The intensity scale represents counts per pixel in the MAD, proportional to $d^{2} \sigma / d \theta_{\text {c.m. }} d M_{R}$. 


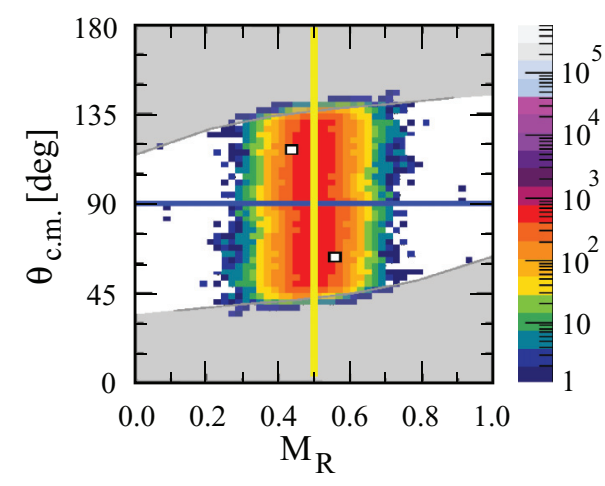

FIG. 3. (Color online) Measured mass-angle distribution for the ${ }^{16} \mathrm{O}+{ }^{196} \mathrm{Pt}$ reaction, expected to lead to fusion-fission. The white squares illustrate how the MAD is populated at two points for each binary event measured. For fusion-fission, the distribution is expected to be symmetric in angle about the blue line, and symmetric in mass about the yellow line. Elastic events are not seen for the ${ }^{16} \mathrm{O}$ beam as they were below the MWPC timing thresholds (see text).

of the fission-like components of the MAD. The software gates applied in generating all the MAD are those shown in Figs. 11(b) and 12.

The second instrumental effect involves target nucleus recoils following elastic scattering, and is relevant to those reactions involving beams heavier than ${ }^{12} \mathrm{C}$ and ${ }^{16,18} \mathrm{O}$. Kinematic considerations show that as the elastic scattering angle of beam particles approaches $0^{\circ}$, the corresponding target recoils are ejected from the target with laboratory angles approaching $90^{\circ}$ and energies approaching zero. Clearly there is an energy threshold below which the recoils will not penetrate the gas window and cathode foil. Thus, they will not appear in coincidence with the corresponding elastic scattering event, and will consequently be absent from the MAD. This is why the elastic-recoil coincidences corresponding to forward angle elastically scattered beam particles are absent or suppressed in the MAD, principally for the $\mathrm{Mg}$ and $\mathrm{Si}$ beams, for which the energies of the recoils at a given angle are low. The distribution with angle of fission-like events in the MAD spectra show that this instrumental low-energy threshold has essentially no effect on the mass-angle distribution of the fission and quasifission events, since unlike target recoils, their laboratory energies do not approach zero.

\section{B. Reaction outcome features}

Having covered the instrumental influences on the experimental MADs, the physical mechanisms responsible for the appearance of each MAD can be explored. An idealized diagram of the features seen in an experimental MAD is presented in Fig. 4. It shows the location in mass and angle of the different reaction outcomes, and also illustrates the origin of the correlation between the mass and angle seen in the quasifission events. Each of these features is discussed below.

\section{Quasielastic and deep inelastic collisions}

The two green shaded regions marked QE in Fig. 4 are mainly populated through elastic and inelastic scattering of the

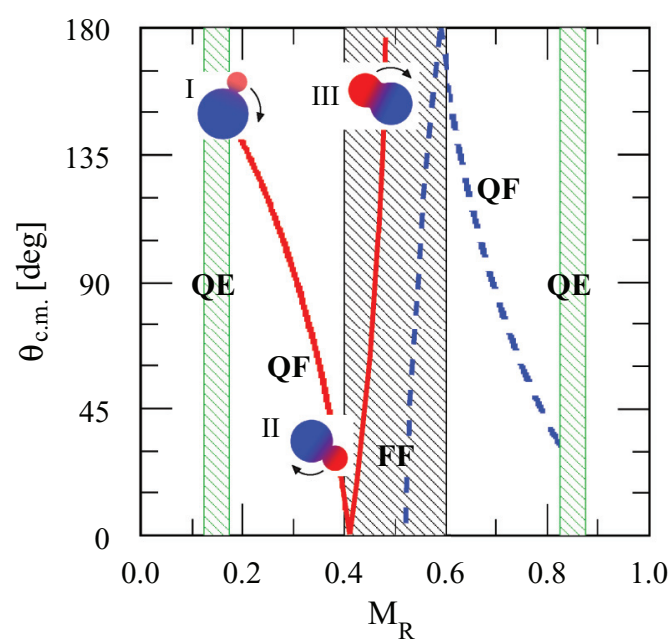

FIG. 4. (Color online) Schematic illustration of the MAD showing the regions corresponding to different reaction processes. Quasielastic and deep inelastic scattering are denoted by QE, fusionfission by FF, and quasifission by QF. The curved red and blue lines correspond to average quasifission trajectories for a single angular momentum (see text).

projectile and target nuclei, with some contribution from transfer and deep inelastic reactions. By deep-inelastic reactions, we mean that class of events where (i) energy is increasingly dissipated into heating the colliding nuclei until complete damping of relative motion occurs, (ii) mass exchange can occur, but without significant mass drift away from the entrance channel mass-asymmetry [36,37], and (iii) deviations from Coulomb trajectories occur due to nuclear orbiting. The angular dependence of the elastic and quasielastic scattering yield essentially follows Rutherford scattering, except at the backward angles, where scattering events are depleted, being transformed into fission-like events as a result of capture, sticking, and mass flow towards symmetry. For elastic scattering, the expected mass ratio $M_{R}$ should correspond to the value expected from the entrance channel projectile and target masses $m_{p}$ and $m_{t}$; i.e., $M_{R}=m_{p} /\left(m_{p}+m_{t}\right)$ if the projectile is detected in MWPC1, or $M_{R}=m_{t} /\left(m_{p}+m_{t}\right)$ if the target nucleus reaches MWPC1.

\section{Fusion-fission and quasifission}

The processes of interest in this work are quasifission and fusion-fission. Both result in fission fragments consistent with a two-body full-momentum transfer (FMT) fission event [9]. Most kinematic properties of the fission fragments resulting from these two processes will be similar, if not identical. However, heavy ion fusion-fission generally shows the peak yield at mass symmetry $\left(M_{R}=0.5\right)$, and a standard deviation in $M_{R}$ of $\sim 0.05$ to 0.08 . Low-energy mass-asymmetric actinide fission typically has a peak mass yield around $A=139$; thus the mass ratio is typically not more asymmetric than $M_{R}=0.60 / 0.40$. The gray shaded fusion-fission band (marked FF in Fig. 4) thus is drawn as extending from $M_{R}=0.4$ to 0.6 . Although both fusion-fission and quasifission can contribute to this shaded region, quasifission alone gives significant population to the 
regions marked QF, located between the quasielastic region and the fusion-fission region. Because the contribution to the MADs of fusion-fission and quasifission overlap, they will be discussed together.

Fusion-fission and quasifission are conceptually distinct in terms of the physical processes driving each outcome. In fusion-fission, the two nuclei fuse and form a compact compound nucleus. The direction and mass of the projectile is by definition "forgotten" through forming a compact equilibrated compound nucleus. Generally after a considerable delay [38], fusion-fission can occur, between several $10^{-20}$ and $\sim 10^{-16}$ seconds later. In quasifission, the system breaks apart before reaching compact equilibrium shapes, often occurring in less than $10^{-20}$ seconds $[2,34]$. This difference in mechanism and timescale can result in clearly observable differences between quasifission and fusion-fission contributions in the MAD.

In fusion-fission, the loss of memory and longer timescale (typically resulting in many rotations before fission) means that no correlation between the fusion-fission fragment masses and angles can be present. Fusion-fission generally shows a maximum yield at symmetric mass splits, and is thus restricted to a band around $M_{R}=0.5$, corresponding to the gray hatched region.

The distinct characteristic of fast quasifission in the MAD arises from two almost independent processes occurring after capture: (1) rotation, resulting from the angular momentum introduced in the collision, and (2) gradual mass equilibration between the projectile- and target-like nuclei, moving asymptotically towards symmetry $\left(M_{R}=0.5\right)$ [2]. Depending on the entrance channel, the dinuclear system may break up soon after capture, or may stick together for more than one rotation. As long as the projectile-like and target-like nuclei break apart within half a rotation, quasifission events show a strong correlation between mass ratio and angle.

With this picture of the quasifission process in mind, three different categories of MAD have been defined. These categories, individually discussed below, will be used to map out how quasifission evolves as a function of entrance channel parameters. The classification assigned to each MAD shown in Fig. 2 is given in Table I. Of course, the MAD characteristics (and associated timescales) vary in a smooth way. However, the following discussion will illustrate that the definition of three categories provides a useful tool for systematic analysis.

\section{MAD1 category}

If scission occurs very soon after initial contact, the system will have experienced little rotation; also little mass will have been able to be exchanged. In such a scenario, a projectile-like fragment is ejected at an angle close to the initial sticking angle. This is illustrated in Fig. 4 as configuration I, with a corresponding target-like fragment at the complementary angle. Such events will populate the regions very close to elastic scattering; in the MAD, they may overlap with events arising from deep-inelastic collisions.

For such short sticking times, the MAD will exhibit a minimum at $M_{R}=0.5$, in sharp contrast to a MAD populated by fusion-fission. This minimum is the defining feature of this class of MAD, which we label MAD1. An example of
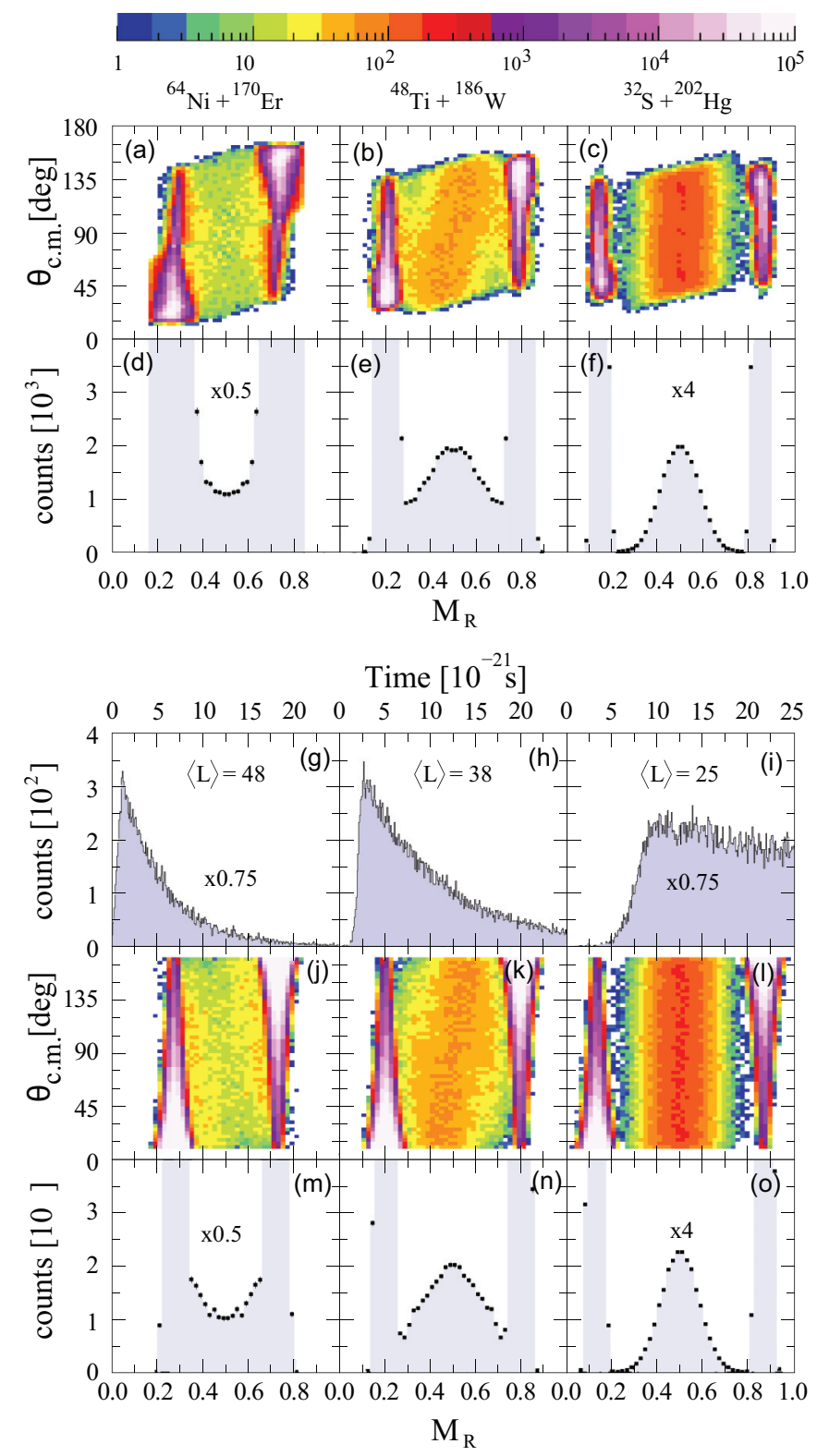

FIG. 5. (Color online) The upper six panels show the experimental MADs in panels (a), (b), (c), and corresponding projections onto $M_{R}$ in panels (d), (e), (f), for the reactions ${ }^{64} \mathrm{Ni}+{ }^{170} \mathrm{Er},{ }^{48} \mathrm{Ti}+{ }^{186} \mathrm{~W}$, and ${ }^{32} \mathrm{~S}+{ }^{202} \mathrm{Hg}$ (see text). The multiplicative factors shown scale the $y$ axis. The lower nine panels show simulated MAD for same reactions and energies in $(\mathrm{g}),(\mathrm{h})$, (i), with the corresponding $M_{R}$ spectra in panels $(m),(n),(o)$, and the sticking time distributions in $(\mathrm{j}),(\mathrm{k}),(\mathrm{l})$. These result in reasonable agreement between the simulations and the measurements shown in the top panels.

this MAD class is shown in Fig. 5 (top left) for the reaction ${ }^{64} \mathrm{Ni}+{ }^{170} \mathrm{Er}$.

\section{MAD2 category}

For quasifission with somewhat longer lifetimes (but still occurring on average before one rotation), the system rotates through a larger angle, and more mass flow is likely, as illustrated in Fig. 4 by configurations II and III. The solid 
(red) line shows (for a single value of angular momentum) the correlated average mass and angle evolution of the projectilelike fragment; the dashed (blue) line displays that of the target-like fragment.

Events that fall in this category of sticking time can be located closer to the elastic scattered events than in fusionfission, and will exhibit a clear nonisotropic distribution in angle. The empirical defining characteristic of MAD2 is an overall mass distribution peaking at $M_{R}=0.5$, and a clear correlation of mass (or mass ratio) with angle. In Fig. 5, an example of this type of MAD can be seen in the top middle panel, for the ${ }^{48} \mathrm{Ti}+{ }^{186} \mathrm{~W}$ reaction.

\section{MAD3 category}

For quasifission events with very long sticking times, greater than that required for the system to undergo one rotation, the nonisotropic distribution with respect to angle will be lost. Fission events will appear close to or inside the fusionfission mass region in Fig. 4, and can be indistinguishable from fusion-fission events. There may be a slight enhancement in the width of the mass distributions where long-lived quasifission is present, in comparison to that resulting from fusion-fission alone [5,29,31]. Thus despite the main distinguishing feature of MADs in this category being the lack of a discernable mass-angle correlation, this does not necessarily imply that no quasifission is present. MADs exhibiting this signature are classified as MAD3. An example of this class is given in the MAD for ${ }^{32} \mathrm{~S}+{ }^{202} \mathrm{Hg}$ shown in the top right panel of Fig. 5.

\section{A quantitative measure of sticking time}

Before presenting a systematic analysis in terms of the MAD classes, an evaluation of the timescales associated with each of the three empirically defined MAD classes will be made, with the help of a simple model of quasifission.

To explore in a quantitative way how different sticking times produce changes in the MAD, a Monte Carlo simulation of mass-angle distributions for quasifission has been developed. This model, based on the physical picture proposed in Ref. [2], and applied in Ref. [34], uses a simple description of mass equilibration and of rotational velocity to determine how quasifission timescales influence MADs. Key inputs include the distribution of sticking times in the system, the timescale for mass equilibration, and the angular momentum distributions following fusion. The last ingredient is obtained from coupled-channels calculations performed using CCFULL [39]. A full discussion of the details of the model can be found in Appendix B.

An attempt to reproduce every MAD in Fig. 2 has not been made. Because the simulation can only provide an estimate of angular velocities without input of time-dependent moments of inertia, it should not yet be used to obtain precise timescales. It can, however, provide a comparative estimate of timescales for each of the three MAD classes. With this in mind, three reactions representing each of the MAD types have been chosen: ${ }^{64} \mathrm{Ni}+{ }^{170} \mathrm{Er}$ for MAD1, ${ }^{48} \mathrm{Ti}+{ }^{186} \mathrm{~W}$ for MAD2, and ${ }^{32} \mathrm{~S}+{ }^{202} \mathrm{Hg}$ for MAD3.
Both the observed and calculated MADs and mass distributions are shown in Fig. 5, with the observed quantities in panels (a) to (f) and the calculated quantities in panels ( $g$ ) to (o). The distributions of sticking times used in each calculation are shown, as are the CCFULL calculated mean angular momenta for each reaction following capture.

For ${ }^{64} \mathrm{Ni}+{ }^{170} \mathrm{Er}$, the MAD is reproduced with a very short mean sticking time: $\tau_{\mathrm{MAD} 1}<5 \times 10^{-21} \mathrm{~s}$. In the case of ${ }^{48} \mathrm{Ti}+{ }^{186} \mathrm{~W}$, a mean sticking time of $\tau_{\mathrm{MAD} 2} \sim 10 \times 10^{-21}$ $\mathrm{s}$ reproduces the experimental MAD. Finally, a lifetime corresponding to $\tau_{\mathrm{MAD} 3} \gg 10 \times 10^{-21} \mathrm{~s}$ provides a good match to the observed MAD for ${ }^{32} \mathrm{~S}+{ }^{202} \mathrm{Hg}$, having a very small fraction of fission before $10 \times 10^{-21} \mathrm{~s}$.

The sticking time distributions used to reproduce the observed MAD for ${ }^{64} \mathrm{Ni}+{ }^{170} \mathrm{Er},{ }^{48} \mathrm{Ti}+{ }^{186} \mathrm{~W}$, and ${ }^{32} \mathrm{~S}+{ }^{202} \mathrm{Hg}$ are consistent with those deduced for the three similarly categorized reactions presented in Ref. [34]. It is thus reasonable that these timescales should provide a representative measure of the reaction timescales for all reactions assigned to the three MAD classes.

\section{MAD classes in the MAD map}

With the three introduced MAD classes, it is now possible to return to Fig. 2 and trace how average quasifission timescales evolve as a function of entrance channel parameters.

Not surprisingly, it can be clearly seen that MAD1 class reactions are found at the upper right, MAD3 in the lower left, and MAD2 in the region between. This trend can be clearly illustrated by considering sets of reactions where the projectile mass is increased while bombarding the same target. A good example can be seen by following the MAD trends for reactions on $\mathrm{Pb}$. For the light projectiles $(\mathrm{O}$ and $\mathrm{S})$, the measured MADs correspond to a long reaction lifetime, consistent with MAD3 quasifission and/or fusion-fission. When moving to heavier projectiles such as Ti, the MADs reveal an obvious correlation between mass and angle, indicating a shorter mean lifetime, with a significant fraction of events arising from quasifission of class MAD2. Moving to the even heavier projectile $\mathrm{Ni}$, mass-symmetric fission events are the least likely, and fast quasifission (MAD1) and deep inelastic reactions dominate. The mean sticking time gets shorter with increasing projectile mass, consistent with earlier measurements [34].

Similarly, the same trend, MAD3 $\rightarrow$ MAD2 $\rightarrow$ MAD1, corresponding to shorter and shorter sticking times, is observed in reactions using the same projectile on heavier and heavier target nuclei. The full transition from MAD3 to MAD1 is seen in Fig. 2 for reactions with ${ }^{48} \mathrm{Ti}$. The reaction with $\mathrm{Sm}$ shows no mass-angle correlation, corresponding to MAD3. When bombarding $\mathrm{U}$, a strong mass-angle correlation is present, and the mass yield shows a minimum at symmetry, corresponding to MAD1.

The same transition is seen over a small range of entrancechannel conditions when producing the same compound system with different projectile-target combinations, moving quickly from MAD3 $\rightarrow$ MAD2 $\rightarrow$ MAD1 as the projectile $Z_{p}$ increases. This can be observed in Fig. 2 for reactions 
forming isotopes of ${ }_{96}^{234} \mathrm{Cm}$, a sequence of reactions recently analyzed in detail in Ref. [33]. The MAD for the reaction of ${ }_{16}^{32} \mathrm{~S}$ with ${ }_{80}^{202} \mathrm{Hg}$ shows no mass-angle correlation, corresponding to MAD3. With increasing projectile $Z_{p}$ and decreasing target $Z_{t}$ the lifetime becomes shorter, and we obtain a distribution consistent with MAD1 for the reactions of ${ }_{28}^{64} \mathrm{Ni}$ with ${ }_{68}^{170} \mathrm{Er}$.

The empirical observation of systematic changes in the MAD as a function of the projectile, target, and compound nucleus atomic number raises the question as to whether a single scaling parameter can describe this systematic behavior.

\section{Effective fissility curves in the MAD map}

The probability of quasifission should be associated with the balance between the repulsive Coulomb and attractive nuclear forces during the reaction. The effective fissility in the entrance channel [40] provides a quantitative measure of this balance at the early stages of the reaction. In Fig. 2, curves of constant effective fissility have been plotted. Details of the effective fissility parameter $x_{\text {eff }}$, as well as the procedure for generating the curves, are described in Appendix C.

If the effective fissility were the only determinant of the reaction outcome, it would be expected that reactions with the same $x_{\text {eff }}$ would show the same pattern in the MAD, and that the threshold for transitions between MAD classes would occur at the same value of fissility. The comparison of experimental MAD with lines of constant $x_{\text {eff }}$ in Fig. 2 shows fair agreement with this expectation, but neither is exactly satisfied.

In the following section, we investigate other ways to map the trends of the experimental results, and attempt to find a single parameter that can be used as a predictor of the characteristics of the experimental MAD, with implications for the probability of quasifission, and the suppression of heavy element fusion cross sections.

\section{SYSTEMATIC TRENDS OF MAD CHARACTERISTICS}

The MAD map (Fig. 2) is a simple way to directly represent the systematic trends in experimental mass-angle distributions. Having chosen beam energies 5\%-10\% above the capture barrier, smooth trends in the characteristics of the MAD with projectile and target (or compound nucleus) atomic number are very evident. It has already been established in previous publications $[13,25,26]$ that such smooth behavior is not necessarily the case at near- and sub-barrier energies, where effects of nuclear structure are undoubtedly much more important. Thus at lower beam energies a similar map would not exhibit such smooth trends everywhere.

For the present set of above-barrier data, the almost linear dependence of the MAD characteristics on the atomic numbers of the projectile and target nuclei suggest that it should be possible to linearize the trends in the MAD using one or two relatively simple ordering parameters.

\section{A. Ordering of MAD by charge product}

The simplest ordering, involving the fewest variables, is discussed first. This mapping, closely related to the MAD map in Fig. 2, is shown in Fig. 6. On the horizontal axis

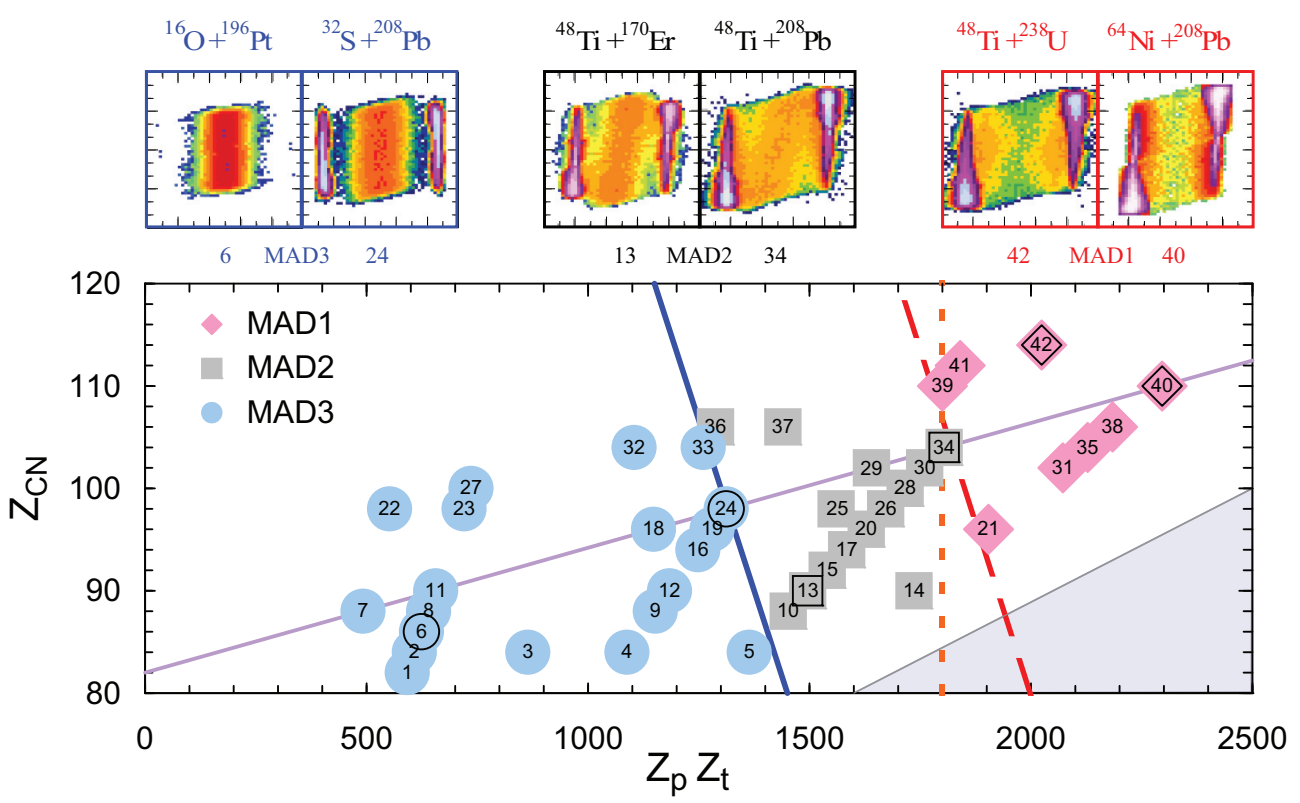

FIG. 6. (Color online) The numbers in the figure refer to the reaction number in Table I, plotted against entrance-channel charge product and compound atomic number. The colored symbol surrounding each number identifies the classification of the experimental MAD, as indicated in the legend at the lop left. The gray shaded region at the bottom right indicates where no reactions are possible. The thin purple line is the locus of reactions with $\mathrm{Pb}$. The diagonal full blue line represents the empirical boundary between reactions with no mass-angle correlation (left) and those that have (right). The dashed and dotted red lines indicate the uncertainty in the boundary of reactions which no longer exhibit a symmetric peak in the angle-integrated fission mass distribution (see text). Examples of MAD are shown in the panels above, identified by their reaction number. 
is the charge product $Z_{p} Z_{t}$, related to the Coulomb energy in the entrance channel. The $y$ axis is the atomic number of the compound system $Z_{\mathrm{CN}}$, the most important variable determining the fissility of the compound nucleus. These axes allow any reaction to be very simply located on the plot. The numerical identifier (see Table I) of each reaction measured in this work is given inside a color coded symbol; blue circles corresponding to the slowest reaction of MAD class 3, gray squares to the intermediate MAD of class 2 , and pink diamonds to the fastest reactions, of MAD class 1. Two examples from each MAD class are plotted in the upper panels. The reaction identification number of each of these examples is outlined in black on the graph below. In the gray shaded region in the lower right, no reactions are possible. The continuous thin purple line from bottom left to top right corresponds to reactions with isotopes of $\mathrm{Pb}$, which will be discussed in the next section.

Although a higher density of points would be desirable, it is clear that the different MAD classes cluster strongly, and approximate boundaries can be drawn (thick lines) between each class. The boundary where a mass-angle correlation becomes significant (between MAD classes 2 and 3) shows a dependence on both the variables in the graph. It is reasonable that this threshold should depend not only on the entrance channel but also on the identity of the compound nucleus being formed, since long timescales imply the system reached much more compact shapes than the contact configuration. Thus the identity of the compound nucleus should play a role. The boundary for $Z_{\mathrm{CN}} \sim 100(\mathrm{Fm})$ occurs at $Z_{p} Z_{t} \sim 1300$, and assuming a linear dependence on $Z_{\mathrm{CN}}$, the trend of the data is represented by the blue full line. This threshold shows a variation from $Z_{p} Z_{t}=1450 \pm 100$ at $Z_{\mathrm{CN}}=80$, down to $1150 \pm 100$ at $Z_{\mathrm{CN}}=120$.

Turning now to the boundary between MAD classes 1 and 2 , the experimental characteristics of MAD of classes 1 and 2 are quite different (no peak at mass-symmetry for class 1), and thus are hardly subject to uncertainty in the experimental MAD. However, the location of the boundary between MAD classes 1 and 2 is not so well defined because the density of data points is lower in this region. In particular, it is not clear whether the dividing line is dependent on $Z_{\mathrm{CN}}$ or not. The long dashed line results if the same gradient as the class 2 and 3 divider is assumed, while the short dashed line divides the data at $Z_{p} Z_{t}=1800$, independent of $Z_{\mathrm{CN}}$. It would be desirable to obtain more experimental MAD in this region to properly understand the systematic behavior.

The common target and projectile elements used in the measurements populate diagonal lines (with positive slope) in this representation. It is easy to distinguish lines within the figure corresponding to reactions with the same projectile element. For example, the line consisting of reaction numbers $14,21,31,35,38$, and 40 corresponds to reactions with ${ }_{28}^{64} \mathrm{Ni}$. Similar trends can be observed for other projectiles, but naturally each projectile line has a different slope. Where the projectile line crosses the thick blue and dashed/dotted red lines in the figure, it indicates a change in the MAD class.

We now move from the simplest possible mapping of the mass-angle distribution landscape to a mapping where the variables reflect the balance between nuclear and Coulomb forces during the collision. This balance is expected to determine the dynamics of reactions forming very heavy elements.

\section{B. Ordering of MAD by fissility parameters}

Reference [41] discussed in detail the expectation of scaling behavior within the context of the schematic "chaotic regime dynamics" model of fusion of heavy nuclei. This is the model [35] that predicted the "extra push" and "extra-extra-push" kinetic energies needed to overcome the conditional and unconditional saddle-point energies, respectively. The successful scaling of calculated "extra-extrapush" energies, and the highlighting of associated landmark saddle-point configurations in the dynamical shape evolution, provides a background and motivation to attempt to order the experimental MAD outcomes in terms of (i) the effective fissility parameter of the entrance channel, $x_{\text {eff }}$, and (ii) the fissility parameter of the compound nucleus, $x_{\mathrm{CN}}$. Unlike the atomic numbers used in previous mapping, each variable has a quantitative interpretation [41], being a measure of the balance between the repulsive Coulomb force and the attractive nuclear force. The former applies to necked shapes close to the (generally) mass-asymmetric contact configuration in the entrance channel, while the latter applies to shapes without a constricted neck [35], and where the mass-asymmetry degree of freedom is not constrained. The equations used to calculate $x_{\mathrm{eff}}$ and $x_{\mathrm{CN}}$ are given in Appendix C, and are tabulated for each reaction in Table I.

Using the same format as Fig. 6, the data are plotted in Fig. 7(a) against these two fissility parameters. In this representation the mass numbers (neutron numbers) of the colliding nuclei play a role in calculating both fissility parameters, unlike in the previous mappings. However, as might be expected from the smaller effect of neutron number compared to proton number in determining fissilities, the data still cluster in the three classes in a similar way to that seen in Fig. 6.

The boundary across which a mass-angle correlation becomes significant (between MAD classes 3 and 2) again shows a dependence on both variables, with a stronger dependence on the entrance-channel than on the compound nucleus fissility. The full blue line is our estimate of this boundary based on the current data. Mass-angle distributions for reactions on this line should show similar mass-angle correlations, associated with similar reaction trajectories and timescales. The same should be true for reactions on nearby parallel lines. The equation of this boundary line is $0.75 x_{\mathrm{eff}}+0.25 x_{\mathrm{CN}}=0.68$, giving $x_{\mathrm{eff}}$ three times the weight of $x_{\mathrm{CN}}$.

It is notable that the weightings of $x_{\mathrm{eff}}$ and $x_{\mathrm{CN}}$ are inverted from those of Ref. [41] that gave the best scaling for the calculated "extra-extra-push" energies required to overcome the unconditional (fission) saddle point and result in fusion. This raises the question of whether there is indeed a simple relationship between the different MAD classes that we have defined, and the "landmark" unconditional and conditional saddle points in the PES. This will need further investigation through theoretical model calculations.

As also seen in Fig. 6, the boundary between MAD classes 1 and 2 is not well-defined because of the lower data density. 

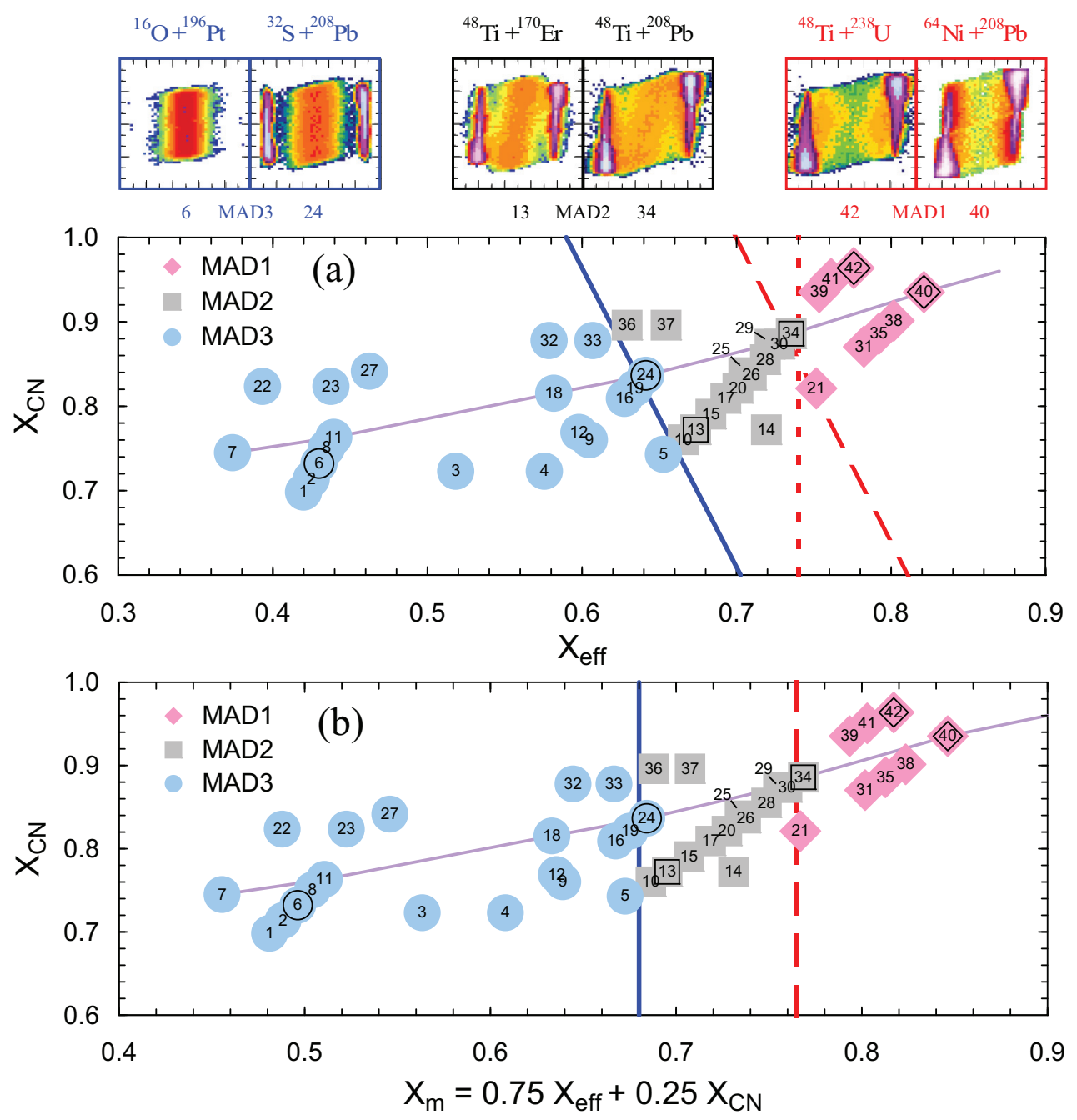

FIG. 7. (Color online) As in Fig. 6, but here plotted (a) as a function of the effective fissility parameter in the entrance channel $x_{\text {eff }}$ and the compound nucleus fissility parameter, and (b) as a function of the mean fissility parameter $x_{\mathrm{m}}$ required to straighten the MAD class boundaries (see text), and the compound nucleus fissility parameter.

A line (red dashed line) with the same slope as the class 2-3 boundary or a line independent of $x_{\mathrm{CN}}$ (red dotted line) could both describe the existing experimental data. According to our definition of MAD1 class reactions, the mass distribution does not peak at symmetry. The limited relaxation towards symmetric mass splits, and the short timescales inferred from our simulations that reproduce the experimental MAD, imply that after contact, the average shape trajectory never reaches close to the compact compound nucleus shape. It would be reasonable that the compound nucleus fissility $x_{\mathrm{CN}}$ should have a smaller influence in determining the boundary between MAD1 and MAD2, compared to that between MAD2 and MAD3, where the quasifission shows mass yields peaked at symmetry, and sticking times at least twice as long.

By defining a weighted mean of the two fissilities, as in Ref. [35], a single mean fissility parameter $x_{\mathrm{m}}$ can be defined by $x_{\mathrm{m}}=0.75 x_{\mathrm{eff}}+0.25 x_{\mathrm{CN}}$, such that the characteristics of experimental MAD (at least of class 2 ) should be very similar for the same $x_{\mathrm{m}}$. Figure 7(b) plots the class of the experimental MAD as a function of $x_{\mathrm{m}}$ and $x_{\mathrm{CN}}$. The class 2-3 boundary depends only on $x_{\mathrm{m}}$, occurring at $x_{\mathrm{m}}=0.68$. The class $1-2$ boundary may not be expected to occur at a constant value of $x_{\mathrm{m}}$ as defined with the $x_{\mathrm{eff}}$ and $x_{\mathrm{CN}}$ weightings given above. Thus, the red dashed line representing this boundary at $x_{\mathrm{m}}=0.765$ can only be considered preliminary at this stage.

\section{Residual modulations due to nuclear structure}

There may be remnant nuclear structure effects (such as those described in Refs. [13,25]) perturbing the smooth systematic trends of the MAD characteristics. This is despite choosing MAD measurements at above-barrier beam energies, which reduces nuclear structure effects known to be important at below-barrier energies. These effects are discussed in the next section.

\section{Magic numbers in the entrance channel}

In Figs. 7(a) and 7(b), as in the previous mapping, the thin full purple lines define the locus of reactions with $\mathrm{Pb}$. 


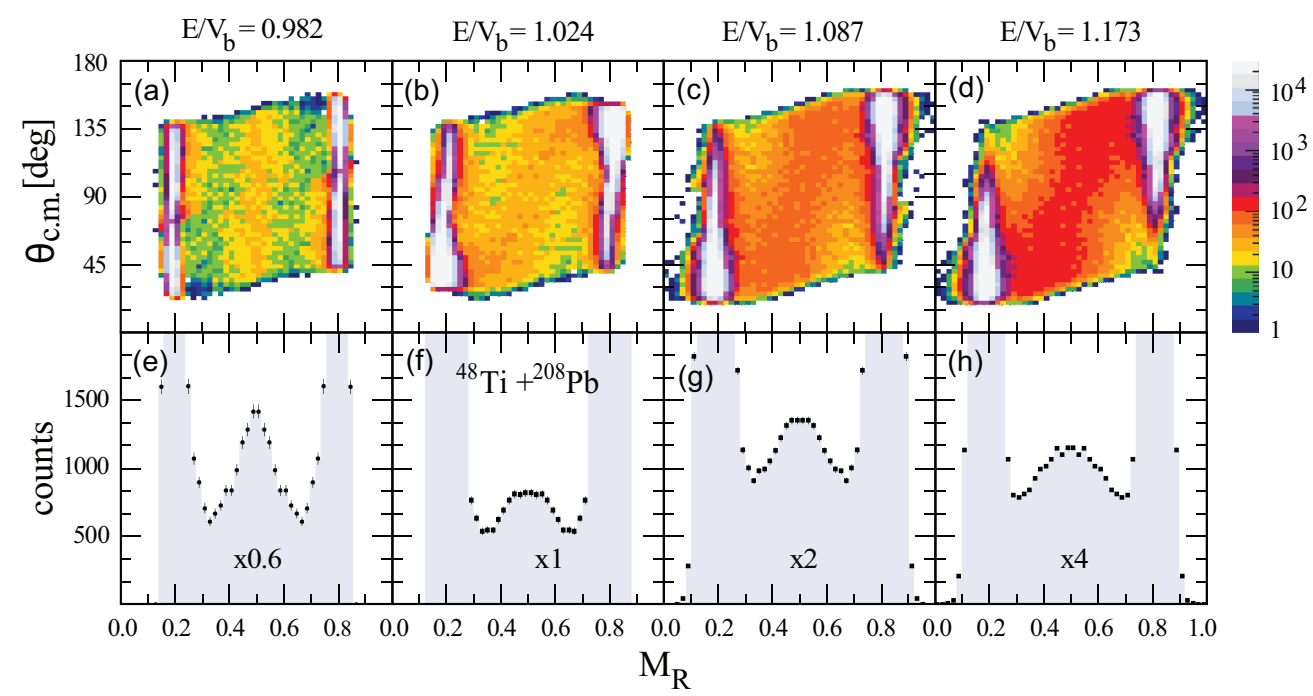

FIG. 8. (Color online) Panels (a) to (d) show the experimental MAD, and panels (e) to (h) the corresponding projection onto $M_{R}$ for ${ }^{48} \mathrm{Ti}+{ }^{208} \mathrm{~Pb}$ reactions at $E / V_{B}$ ranging from 0.98 to 1.17 . The mass width and the correlation between mass and angle both increase rapidly as the beam energy increases across the barrier energy, signifying the damping with energy of the effects of closed shells that appear to assist fusion in this reaction.

The current data set includes only reactions with the doubly magic ${ }^{208} \mathrm{~Pb}$. Reactions 24 and 34 , with ${ }^{208} \mathrm{~Pb}$ as the target nucleus, deviate most from the attempt to parametrize the MAD systematics using a single variable $x_{m}$. Reaction 34 $\left({ }^{48} \mathrm{Ti}+{ }^{208} \mathrm{~Pb}\right)$ appears to intrude into the region of MAD class 1. This is consistent with a previous study showing the beam energy dependence of the angle-integrated mass widths [32] for ${ }^{48} \mathrm{Ti}$ reactions on targets from $\mathrm{Sm}$ to $\mathrm{Pb}$. There it was found that the mass width for ${ }^{48} \mathrm{Ti}+{ }^{208} \mathrm{~Pb}$ only reached that for reactions with neighboring nonmagic target nuclei well above the capture barrier energy, at $E / V_{B} \sim 1.15$.

Figure 8 shows the MAD in panels (a) to (d) and projected mass-ratio spectra in panels $(\mathrm{e})$ to $(\mathrm{h})$ for this reaction, for beam energies from below barrier to well above barrier, including that at $E / V_{B}=1.09$ presented in Fig. 2. There is a strong evolution with beam energy. Following the interpretation of Ref. [13], the relatively narrow $M_{R}$ distribution and weak mass-angle correlation at the lowest beam energy is attributed to closed shells in the target nucleus allowing greater interpenetration of the two nuclei following capture. Even in the above-barrier energy range selected, there is an indication that magic numbers in the entrance channel still affect the mass width and MAD shape, thus implying a difference in the sticking timescale. From this conclusion it would be reasonable to infer an enhancement in the probability of fusion through an increased value of $P_{\mathrm{CN}}$.

These results highlight the importance of selecting measurements at above-barrier energies to investigate smooth trends in reaction dynamics, with minimal effects of nuclear structure complicating the picture.

\section{Static deformation in the entrance channel}

Reaction $24\left({ }^{32} \mathrm{~S}+{ }^{208} \mathrm{~Pb}\right)$ is the second of the two noted reactions involving ${ }^{208} \mathrm{~Pb}$. This shows no evidence of any mass-angle correlation in the MAD, within experimental uncertainty, and is thus completely consistent with MAD class 3. However, it is flanked by reactions of class 2 at both smaller and larger values of $x_{\mathrm{CN}}$, showing clear evidence of a considerable mass-angle correlation. A similar increase in sticking time to that inferred for ${ }^{48} \mathrm{Ti}+{ }^{208} \mathrm{~Pb}$ might well also occur in this reaction. However, without a mass-angle correlation there is a much smaller possibility of observing a significant change with beam energy of the MAD and the mass width, as was seen so clearly for the ${ }^{48} \mathrm{Ti}+{ }^{208} \mathrm{~Pb}$ reaction.

We must also consider whether these neighboring reactions might also exhibit residual effects of entrance-channel nuclear structure. They are ${ }^{48} \mathrm{Ti}+{ }^{162} \mathrm{Dy}$ (reaction 10 ) and ${ }^{28} \mathrm{Si}+{ }^{238} \mathrm{U}$ (reaction 36). Both target nuclei have a large static prolate deformation. Both reactions show an increased mass width [32] and mass-angle correlation [42] at sub-barrier energies.

This is illustrated in Fig. 9 for the ${ }^{28} \mathrm{Si}+{ }^{238} \mathrm{U}$ reaction. In this case, the MADs and projected $M_{R}$ spectra range in energy from $E / V_{B}=0.92$ to $E / V_{B}=1.12$, including the data at $E / V_{B}=1.05$ shown in Fig. 2. At the lowest energy, the data show two discrete components in both mass and angle-a fast mass-asymmetric component [arrowed in panel (a)] and a slower component peaked at mass symmetry. As the beam energy increases, the fraction of the fast component decreases markedly.

This phenomenon has been attributed to the effect of deformation alignment. At sub-barrier energies, only collisions with the deformation-aligned target nucleus lead to capture and dissipative reactions. The resulting elongated capture configuration was first proposed in Refs. [8,9,43] as an explanation for the evidence in the measured fission properties of inconsistency with the expectations for fusion-fission. Similar results and conclusions have since been found for many other reactions [10-12,44]. At above-barrier energies, all orientations contribute to the observed fission events. 


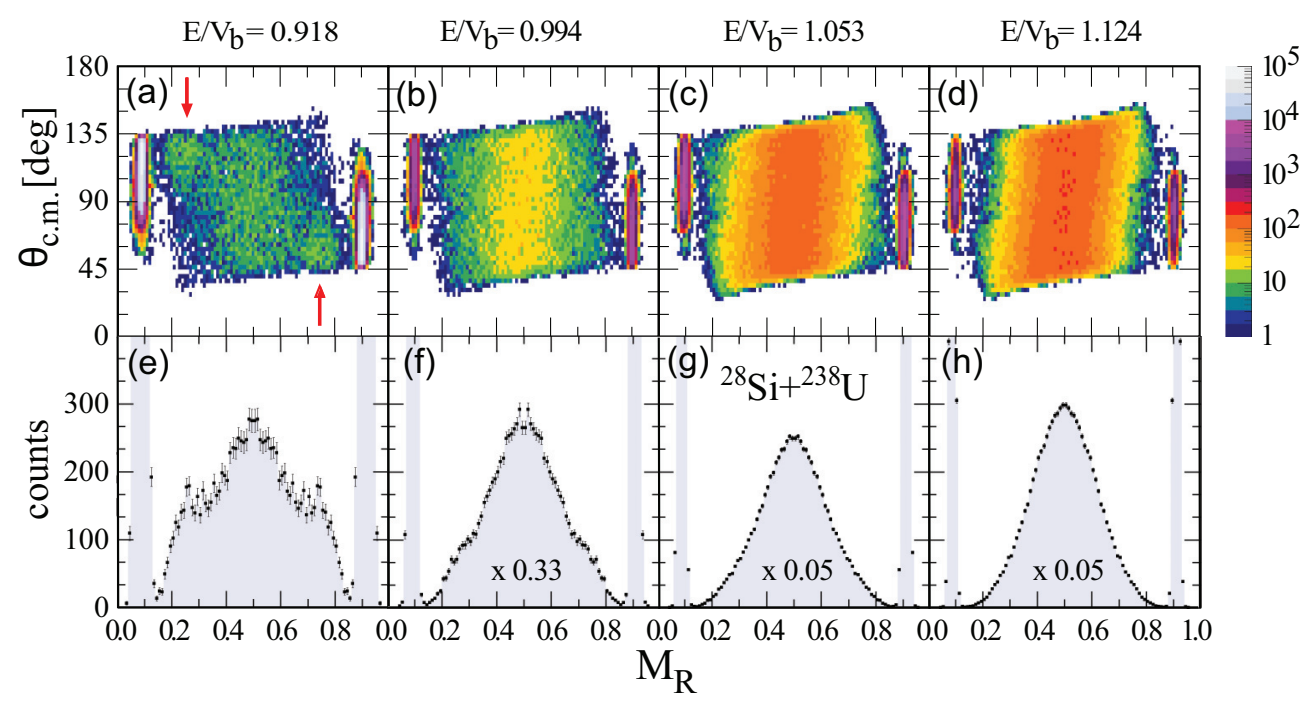

FIG. 9. (Color online) Panels (a) to (d) show the experimental MAD and (e) to (h) the corresponding projections onto $M_{R}$ for ${ }^{28} \mathrm{Si}+{ }^{238} \mathrm{U}$ reactions at $E / V_{B}$ ranging from 0.92 to 1.12 . The red arrows indicate the fast mass-asymmetric quasifission associated with deformation-aligned collisions.

However, the deformation-aligned (tip) collisions are still present at above-barrier energies, and may make a significant contribution to the observed mass-angle correlation. If the target nucleus did not have a large static deformation, it may be that a mass-angle correlation would not be so evident in the experimental MAD.

\section{Shell structure near scission}

The final nuclear structure effect that can influence MADs is shell structure in the potential energy surface closer to the exit configuration. In spontaneous and low-energy fission of actinide isotopes, shell effects in the potential energy surface result in the well-known systematic evolution with mass and charge of the prominent asymmetric fission mass distributions. Despite the higher excitation energies in heavyion reactions, experimental mass distributions have provided evidence that has been interpreted $[10,11,45,46]$ as showing that mass distributions could be significantly influenced by, or even determined by, shell structures associated with the system closer to the exit channel than to the entrance channel configuration.

The role of doubly magic ${ }^{208} \mathrm{~Pb}$ in the exit channel has become prominent in discussions of both experimental mass distributions [47] and scission time distributions [48], as well as in theoretical calculations [49]. Whether the timescale and value of $P_{\mathrm{CN}}$ are significantly reduced or increased by an energetically favored valley is a question that might be best addressed theoretically. Such a valley at large mass asymmetry could be expected to be more important in heavy-ion reactions than it is in low-energy fission. The system starts with a large elongation, and with a larger mass asymmetry. Thus when evolving in mass asymmetry it might be expected to "fall into" such a valley, leading to scission.

The peaked yield of fast mass-asymmetric quasifission seen for the ${ }^{28} \mathrm{Si}+{ }^{238} \mathrm{U}$ reaction (red arrows in the leftmost panel of Fig. 9) is associated with deformation-aligned collisions. The mass distribution may result from such a feature in the PES. The yield of this component peaks at $M_{R}=0.26 / 0.74$, corresponding to mass splits 70/196. This feature may be consistent with the extra stability associated with proximity to the doubly magic ${ }^{208} \mathrm{~Pb}$. However, there may be dynamical effects playing a role in determining the mass distribution. One such effect is sequential fission of heavy quasifission products [50] having masses between that of the actinide target nucleus and nuclei in the less-fissile region below ${ }^{208} \mathrm{~Pb}$. Such a reduction in yield could contribute significantly to the observed mass distribution. Further experimental studies should be able to resolve different potential contributions to these and similar observations.

Current Langevin model calculations associate peaks seen in the quasifission mass yields for similar reactions [11] with a valley in the PES. However, in modeling nuclear structure effects, the interplay between the Coulomb field, favoring elongated shapes, and the spherical shell-stabilized ${ }^{208} \mathrm{~Pb}$ may be difficult to address in dynamical models where the shape degrees of freedom are too restrictive.

\section{IMPLICATIONS FOR FUTURE HEAVY ELEMENT STUDIES}

The nuclear structure effects discussed in the previous sections highlight the challenges in developing a model of heavy element fusion reactions addressing all these effects fully dynamically. They also provide some limitation to the completely empirical investigation that we have made of the underlying systematic behavior of mass-angle distributions. However, it is important to recognize that the nuclear structure effects only provide small and local modulations to the otherwise smooth changes in quasifission characteristics seen in our measurements at beam energies $\sim 6 \%$ above the capture barrier. 


\section{A. Using MADs to validate dynamical models}

The ultimate goal is to obtain experimental data to guide the development of - and also to validate-realistic dynamical models of heavy element fusion that reproduce all the relevant features seen in experiments.

These features should include

(i) heavy element yields;

(ii) mass-angle distributions;

(iii) mass-energy distributions;

(iv) pre-scission and post-scission multiplicities and angular correlations of evaporated particles;

(v) scission time distributions.

Calculating all these properties would make a heavy demand on any model. This is why we believe that it is desirable to validate models at the simplest level first, before including more complex physical effects. The present data set provides a playground for theoretical calculations where nuclear structure effects are a perturbation on the landscape, rather than the main drivers [13]. Other data sets, at lower bombarding energies, can show the opposite behavior. The appropriate data sets must then be chosen to test different aspects of dynamical models.

\section{B. Perspective on further MAD measurements}

From an experimental perspective, several steps still have to be taken to realize the full potential of MADs in probing quasifission. These include

(i) experimental isolation of the effect of angular momentum on timescales;

(ii) experimental confirmation of the mass-equilibration time constant;

(iii) investigation of the role of neutron richness in quasifission dynamics independent of shell effects;

(iv) more extensive MAD measurements to study the effects of magic numbers at and below the capture barrier energy;

(v) extension of measurements into the angular region currently with no coverage, to obtain total mass yields.

These questions should be addressed in future MAD measurements. Refinement of the Monte Carlo MAD simulation model should help to address the first two questions, but the ultimate goal is that sophisticated dynamical models should calculate MADs directly, which can then be compared quantitatively with the corresponding experimental MADs.

\section{Importance of nuclear structure in superheavy element formation reactions}

It is the reactions that are most favorable to fusion that are the most relevant to superheavy element formation. The most successful reactions involve fusion of the doubly magic nucleus ${ }^{48} \mathrm{Ca}$ with actinide nuclei having large static prolate deformations. Ultimately, a good understanding of the dynamics of fusion involving both magic nuclei and statically deformed nuclei must be achieved, to permit the development of models of heavy element fusion with true predictive power.
It would thus be desirable to have a quantitative understanding of why ${ }^{48} \mathrm{Ca}$ is so favorable to heavy element formation. How important is its neutron richness, which results in the formation of neutron-rich compound systems? How important is the fact that it is doubly magic in determining the fusion dynamics and thus $P_{\mathrm{CN}}$ ? Is it the combination of magicity and $N / Z$ ratio that is critical [13]?

The second key question is the role of the static deformation of the heavy partner in the collision. As first proposed from experimental results in Refs. [8,9], and subsequently confirmed through both quasifission [11,51] and ER measurements [12], collisions with the tips of heavy prolate deformed nuclei show a reduced probability of fusion leading to a compact compound nucleus. As a corollary, it was also suggested [8,9] that in collisions with the equator, the fusion probability may be enhanced over that of an equivalent spherical nucleus. It is not yet clear how important this potential enhancement has been in the successful production of superheavy elements. It could also be that there are subtle nuclear structure effects associated with the combination of the doubly magic ${ }^{48} \mathrm{Ca}$ projectile and equatorial collisions. Realistic microscopically based dynamical fusion models may well be required to address the last question.

The answers to these questions will ultimately allow quantitative predictions for reactions with other projectiletarget combinations, and define the future directions of heavy element research.

In order to address these questions, we also need to understand the reaction timescales and outcomes from reactions with fewer or none of these special characteristics. The underlying smooth trends seen in the experimental MAD across a wide range of reactions provide a baseline from which local nuclear structure effects become clearer.

\section{SUMMARY AND CONCLUSIONS}

In this work, we have presented MADs from a wide range of reactions, measured at above barrier energies to minimize effects of nuclear structure. The systematic behavior that has been revealed will help with the development and testing of dynamical models of fission capable of reliably predicting $a$ priori experimental observables for any given reaction forming heavy and superheavy elements.

To predict fusion cross sections for any reaction, we must first understand the dynamics that drive reaction outcomes. The experimental determination of $P_{\mathrm{CN}}$ provides significant insights (though even this is not so easy to achieve). However, the value of $P_{\mathrm{CN}}$ represents an integral of the dynamics all the way from capture, and gives no information on timescales. Thus, $P_{\mathrm{CN}}$ alone can only offer limited information on the dynamical evolution of the dinuclear system across the PES. To probe these dynamics and assess theoretical models, we must choose other means of probing the random walk across the PES. This is where experimental MADs are important.

MADs offer insight into two aspects of the dynamics: (1) the underlying physics related to the changing balance between Coulomb and nuclear forces, which varies smoothly as a function of the numbers of neutrons and proton in the two colliding nuclei, and (2) the fine details introduced by the 
nuclear structure of the projectile and target nuclei. While their influence on the MAD cannot be completely disentangled, we know that nuclear structure effects tend to wash out for reactions measured at beam energies above the capture barrier. By first investigating the systematics of reactions at above-barrier energies, where nuclear structure effects are minimized, we have been able to investigate how the basic properties of the colliding nuclei determines the predominant reaction outcome. Subsequently, a new MAD map should be generated for measurements at below-barrier energies, where nuclear structure effects can be very important.

This work presents a first step, then, towards a more complete and quantitative understanding of nuclear reaction dynamics. The MAD classification maps shown in Fig. 7 provide a simple and powerful tool for experimental research. For investigations of quasifission itself, a consideration of the $x_{\mathrm{eff}}$ and $x_{\mathrm{CN}}$ values will allow the selection of the most useful reactions for study, particularly with regards to explorations of quasifission timescales. When aiming to form isotopes of heavy elements, the data confirm that the most mass-asymmetric reaction is likely to be the most favorable to form a compact compound nucleus.

As more data are obtained, and more comprehensive dynamical models are developed, we will then be able to return to the question we began this work with: how does the $P_{\mathrm{CN}}$ term in Eq. (1) evolve as a function of all reaction parameters? What variables are important in predicting the evolution of the dinuclear system across the PES, and therefore, in understanding $P_{\mathrm{CN}}$ ? These remain difficult questions. The results presented here provide insights into nature that will help in answering these questions, and therefore assist in planning the synthesis of a wider range of superheavy isotopes in sufficient quantity for detailed exploration of their properties.

\section{ACKNOWLEDGMENTS}

The authors are grateful for the long-term contributions of D. C. Weisser, N. Lobanov, T. Kibèdi, and all the accelerator staff of the ANU Heavy Ion Accelerator who have provided the capabilities that have been crucial in pursuing this research program. The authors acknowledge support from the Australian Research Council through Discovery Grants No. DP110102858, No. DP110102879, and No. DP130101569 and Fellowships FL110100098 and FT120100760.

\section{APPENDIX A: EXTRACTING MASS-ANGLE DISTRIBUTIONS}

To reconstruct the characteristics of each event detected in the two large multiwire proportional counters, the position and time information for each pair of coincident fission fragments was converted to obtain the required physical reaction outcome variables as described below.

\section{Converting position to angle}

The time differences between the delay line readouts of each end of the $X$ and $Y$ wire planes could be transformed into position by combining the known dimensions of each detector with data recorded in singles that illuminated each detector's full active area. This relies on the excellent linearity of the MWPC position readout [52]. After gating to exclude the few events appearing outside the physical detector area (that can result from mixing of events from two beam bursts) the $(X, Y)$ positions were converted into scattering angle $\theta$ and azimuthal angle $\phi$. Accuracy of the transformation could be checked through comparing measurements for elastic scattering with the known elastic folding angles in $(\theta, \phi)$.

\section{Obtaining flight times}

Time (and thus velocity) information for each fission fragment was determined in two different ways, depending on whether or not a narrow beam pulse was available for the beam used in the experiment. The two methods used are referred to as the "absolute time method" (ATM) and the "relative time method" (RTM). For the ATM the velocity vectors of both binary reaction partners are determined using their calibrated positions and their individual time-of-flight information. A more detailed description of the ATM used in this work can be found in the Appendix of Ref. [9]. With the RTM the corresponding vectors are determined from the positions and the time difference between the fragments. More details of the RTM used in this work can be found in the Appendix of Ref. [29].

After calibration of the time spectra using an Ortec time calibrator, obtaining accurate mass ratios in the analysis requires the determination of one or two additional time calibration constants, which are determined directly from the experimental data.

The first variable is the time offset $(\delta t)$ between signals from the two detectors, related to time delays through different electronic units and cables. This should not change between measurements for a given electronics setup, and is the only additional time calibration constant required for the RTM analysis. It was determined primarily by requiring that the centroid (before mirroring) of fusion-fission mass distributions should be at $M_{R}=0.5$. The value of $\delta t$ can be confirmed for any measurement by two methods. The first relies on the fact that the mass distribution must be symmetric about $M_{R}=0.5$ at $\theta_{\text {c.m. }}=90^{\circ}$. For many reactions it is easy to check that the nonmirrored $M_{R}$ spectrum peaks at 0.5 for a gate set around $90^{\circ}$. The second method makes use of the known mass ratio for elastically scattered events. In this case, care must be taken that neither the beam particles or the heavy elastic recoils have pulse heights close to the electronic thresholds; otherwise time walk near threshold, even in a constant fraction discriminator, can give a significant perturbation to the time and thus the extracted $M_{R}$.

The second variable, required for the ATM analysis, is the absolute time of arrival $\left(t_{0}\right)$ of the beam pulse on the target with respect to the RF reference, relative to which the detector time information is recorded. This varies from one measurement to another, whenever the beam energy or species is changed. This variable is determined for each measurement by requiring that for binary events (principally fission, but also scattering 
events with pulse heights well above timing thresholds) that the average parallel component of the source velocity in the laboratory frame is equal to the calculated center-of-mass velocity, which is also the velocity of the compound nucleus $v_{\mathrm{cn}}$. The time-of-flight or the time difference between two fission fragments and the position information were then used to determine the individual fragment velocities $v_{i}$.

\section{Mass ratio determination}

The kinematic coincidence method used in the analysis does not allow a direct measure of the mass of each fission fragment. It does permit a reliable measure of the mass ratio of the two fragments [as defined in Eq. (2)]. For this reason, the MAD spectra are presented in terms of the experimental mass ratio, eliminating introduced assumptions and uncertainties relating to particle evaporation before and after scission.

Energy loss corrections were made by first using the reconstructed velocities from either the ATM or RTM methods to make an initial estimate of the fragment masses and kinetic energies, neglecting particle evaporation. Corrections for energy loss suffered in the target by both fragments could then be made. It was assumed that interactions occurred at the center of the target. The corrected velocities were then transposed back to the center-of-mass frame and used to obtain the mass ratio for the fragment recorded in the back detector:

$$
M_{R, 1}=\frac{m_{1}}{m_{1}+m_{2}}=\frac{v_{\mathrm{c} . \mathrm{m}_{.}, 2}}{v_{\mathrm{c} . \mathrm{m} ., 1}+v_{\mathrm{c} . \mathrm{m} ., 2}} .
$$

Here, $v_{\text {c.m., } i}$ is the velocity in the center-of-mass frame for the reaction fragment recorded in the back $(i=1)$ or front $(i=2)$ detector. Naturally, the mass ratio for the fragment measured in the front detector is given by $M_{R, 2}=1-M_{R, 1}$. Through rapidly converging iterations of energy loss correction, final mass ratios and center-of-mass velocities, as well as center-ofmass scattering angles and kinetic energies, were determined for each event.

\section{Event selection criteria}

Once fission fragment velocities and $M_{R, i}$ have been determined for all coincident events, gating is required in order to select out binary (full momentum transfer) events, and eliminate reactions from light impurities in the targets. From this point on in the analysis, each reaction and beam energy was analyzed individually, in order to account for the different reaction mechanisms that are possible for different projectile-target combinations and different beam energies.

To exemplify and illustrate the analysis procedure, as well as display the commonly utilized gates, the gating and analysis of the reaction ${ }^{40} \mathrm{Ca}+{ }^{238} \mathrm{U}$ at $E_{\text {beam }}=240.5 \mathrm{MeV}$ is discussed in detail. The data were recorded using the CUBE spectrometer in geometrical configuration 1, and were analyzed using the ATM.

\section{a. Initial separation of fission events from elastic scattering}

Figure 10 shows a scatter plot of the experimentally determined mass ratio $M_{R}$ vs $v_{\|} / v_{\mathrm{cn}}$ for fragments detected in

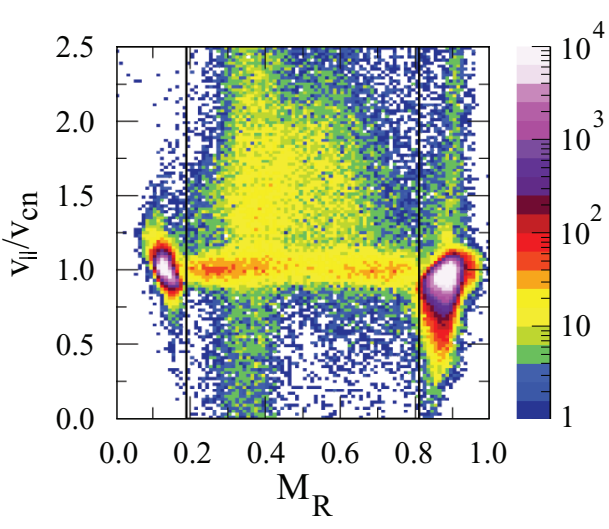

FIG. 10. (Color online) Scatter plot of $v_{\|} / v_{\mathrm{cn}}$ vs $M_{R}$ for fragments detected in the back detector in the reaction ${ }^{40} \mathrm{Ca}+{ }^{238} \mathrm{U}$ at $E_{\text {beam }}=$ $240.5 \mathrm{MeV}$. The rectangular gate (black) is used to select fission events and remove the elastic scattered events. Further details in text.

the back detector, where $v_{\|}$is the extracted velocity component parallel to the beam [9] and $v_{\mathrm{cn}}$ is the calculated velocity of the compound nucleus in the laboratory frame (also the center-of-mass velocity). For a correct time calibration, this ratio is expected to be centered around unity for FMT fission events, which are the events of interest when generating the MAD histograms.

Elastic scattering of the projectile and target nuclei can also be observed in Fig. 10 as the two high-intensity areas near $M_{R} \sim 0.14$ and $M_{R} \sim 0.86$. For reactions with the lighter projectiles ${ }^{12} \mathrm{C}$ and ${ }^{16} \mathrm{O}$, detector electronics thresholds were set to reject elastic events, which are thus not visible. Events populating the region between the two elastic peaks are fission fragments. Using the rectangular gate shown in Fig. 10 (black), fission-like events are selected for defining further gates. The reason for making this selection is made clear below.

\section{b. Separation of binary fission from three-body events}

Besides events arising from binary fission with full momentum transfer (distributed in the band centered at $v_{\|} / v_{\mathrm{cn}}=1.0$ ) a significant number of events with higher $v_{\|}$are also observed in this reaction. These are three-body events associated with reactions resulting in a projectile-like nucleus and two fragments from fission of the target-like nucleus. For the final analysis and the generation of MAD plots, it is crucial to separate these events from the binary fission events of interest. The largest fraction of three-body transfer events was found with reactions using projectiles of ${ }^{40} \mathrm{Ca}$ and ${ }^{48} \mathrm{Ti}$ bombarding the ${ }^{238} \mathrm{U}$ target. The scatter plots discussed below are for the former reaction.

Due to the momentum carried by the recoiling projectilelike nucleus following transfer, both the parallel and perpendicular velocity components $\left(v_{\|}\right.$and $v_{\perp}$ ) of the target-like nucleus when it undergoes scission will generally differ significantly from the full momentum transfer events [9]. This velocity difference provides an excellent event selection criterion to separate the three-body events from the full-momentum transfer binary events.

Figure 11 shows two scatter plots of $\left(v_{\|}-v_{\mathrm{cn}}\right)$ vs $v_{\perp}$, for which data were sorted without [Fig. 11(a)] and with 

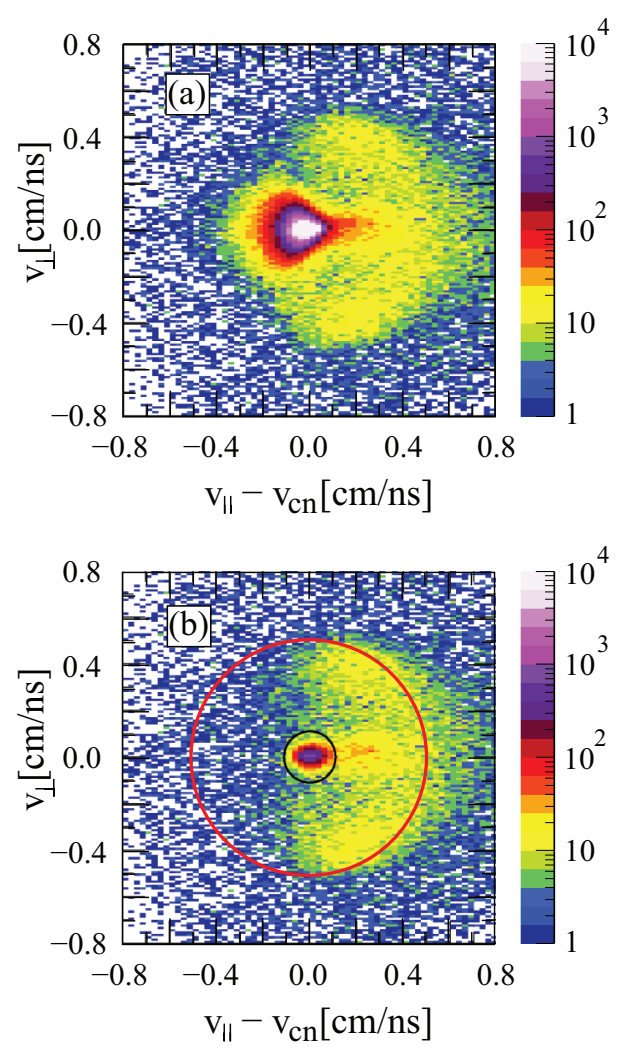

FIG. 11. (Color online) Scatter plots of $\left(v_{\|}-v_{\mathrm{cn}}\right)$ vs $v_{\perp}$ for the reaction ${ }^{40} \mathrm{Ca}+{ }^{238} \mathrm{U}$ at $E_{\text {beam }}=240.5 \mathrm{MeV}$. Panel (a) shows all events, including elastic scattering, whereas (b) required the fission gate shown in Fig. 10. The black circular gate selects full momentum transfer events for generation of the MAD. The red circle has a radius $v_{\mathrm{cn}}$. Most transfer-fission events would be expected to lie inside this circle.

[Fig. 11(b)] the rectangular gate on fission events shown in Fig. 10. Full momentum transfer events of interest are expected to be centered around $v_{\|}=v_{\mathrm{cn}}$ and $v_{\perp}=0$, and are therefore expected to be distributed around $(0,0)$ in the scatter plot. This property is not unique to FMT fission events, as all binary events from the collisions of interest, namely with the heavy nuclei in the target, should be distributed around $(0,0)$. Because of the large angular coverage of the detectors, elastic and quasielastic events are dominant. In the back angle detector, the most intense particle flux is of low-energy target-like recoils associated with forward angle elastic scattering. These can be strongly affected by energy loss and angle scattering in the target and detector foils, giving a much broader distribution than kinematics alone would produce. For this reason, setting an appropriate gate for the FMT fission events in the $\left(v_{\|}-v_{\mathrm{cn}}\right)$ vs $v_{\perp}$ matrix requires that elastic events be excluded. Comparing the two scatter plots, Figs. 11(a) and 11(b), the necessity to remove the elastic scattered events before setting the gate on the FMT fission events is obvious. The full momentum transfer fissions are strongly peaked around $(0,0)$. A tight circular (black) or elliptical gate can then be set around these fission events; this accepts almost all of the FMT binary fission events while at

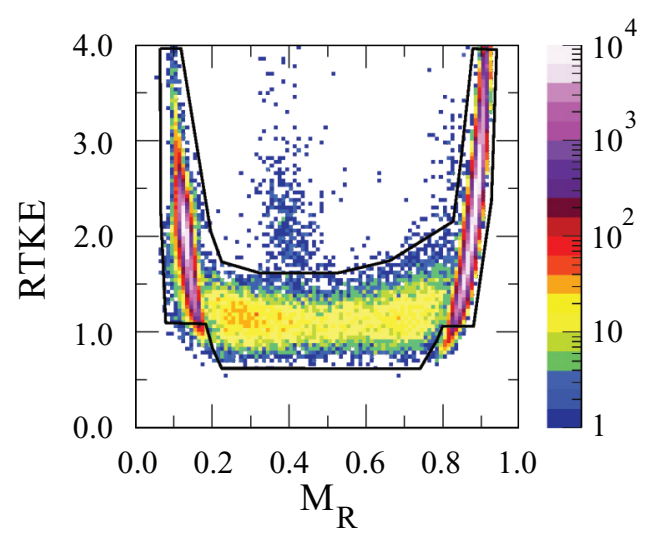

FIG. 12. (Color online) Scatter plot of deduced mass ratio vs relative total kinetic energy for the reaction ${ }^{40} \mathrm{Ca}+{ }^{238} \mathrm{U}$ at $E_{\text {beam }}=$ 240.5 MeV. The black FMT gate shown in Fig. 11(b) has been applied, but not the rectangular gate in Fig. 10. The black polygon is the last gate applied to generate the MAD (see text).

the same time reducing the three-body events very significantly (estimated to be typically cut to $\sim 1 \%$ ).

\section{c. Further reduction of contaminant events}

The final stage of the analysis utilizes the black FMT gate shown in Fig. 11(b) together with the previously described event selection criteria in position and time. From this point in the analysis generating the final MAD, elastic and deepinelastic scattering events are no longer explicitly gated out using the gate shown in Fig. 10. Figure 12 shows the scatter plot of the deduced mass ratio versus relative total kinetic energy (RTKE) [38]. RTKE is defined as $\frac{T K E_{\text {exp }}}{T K E_{V}}$, where $T K E_{\exp }$ is the total kinetic energy determined from the experimental data and $T K E_{V}$ is the calculated most probable total kinetic energy release for fission following Ref. [53], and its extension to asymmetric mass splits [54]. Events with RTKE close to unity correspond to full damping of the initial kinetic energy. This is one of the empirical distinctions between deep-inelastic scattering, where entrance channel kinetic energy need not be fully damped (dissipated), and quasifission, where it is.

FMT fission fragments are found in the horizontal band around RTKE $\sim 1$. Elastic and quasielastic scattering events comprise the high-intensity areas at large RTKE and extreme $M_{R}$. The wide distribution in RTKE in these two intense bands reflects the large number of such events, and the instrumental resolution effects noted above. It is desirable to minimise the contribution of these events within the region of the quasifission events. This is done by introducing the gate shown in Fig. 12 (black polygon), which will remove not only events from the tail of the scattering distributions but also events observed above the FMT fission band, which are probably remnant transfer fission events. As illustrated, the gate rejects a small fraction of the elastic and deep-inelastic scattering events. This, however, is of no significance in generating the MAD scatter plots, where the focus is on the binary fission events. 


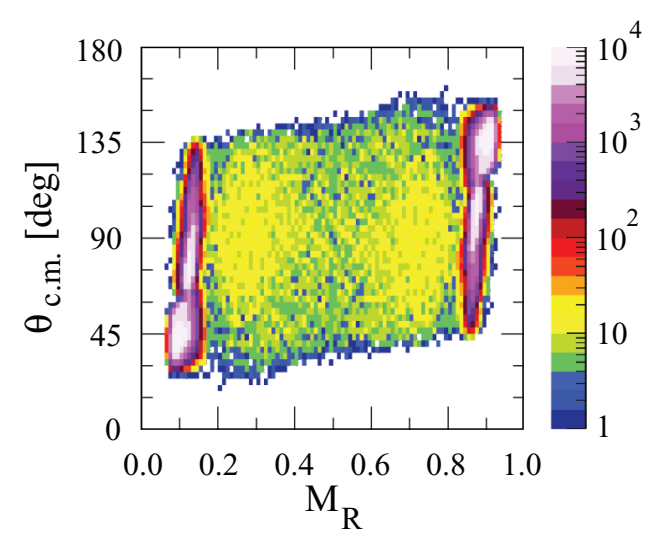

FIG. 13. (Color online) MAD scatter plot for the reaction ${ }^{40} \mathrm{Ca}+{ }^{238} \mathrm{U}$ at $E_{\text {beam }}=240.5 \mathrm{MeV}$.

\section{d. Determination of the mass-angle distributions}

Once the velocity and RTKE conditions shown in Figs. 11(b) and 12 have been applied, the mass ratio $M_{R}$ and center-of-mass scattering angle $\left(\theta_{\text {c.m. }}\right)$ for fragments detected in the back detector can be used to generate the final mass-angle-distribution (MAD) scatter plots. Figure 13 shows the final experimental MAD for the reaction ${ }^{40} \mathrm{Ca}+{ }^{238} \mathrm{U}$ at $E_{\text {beam }}=240.5 \mathrm{MeV}$, where the event selection criteria described above have been applied. The geometrical acceptance of the detector is about $100^{\circ}$ in $\theta_{\text {c.m. }}$, independent of mass ratio. The angular coverage shifts with mass ratio by about $15^{\circ}$.

The vertical bands around $M_{R} \sim 0.14$ and 0.86 are principally from quasielastic events, associated with projectile-like and target-like nuclei, respectively. Fission-like events are clearly seen in the region between the two elastic bands. They show a strong correlation between mass and angle, indicating the short timescale of this reaction.

\section{APPENDIX B: QUASIFISSION SIMULATION}

The basis of the Monte Carlo model developed [34] to simulate quasifission mass-angle distributions is shown graphically in Fig. 14. The figure shows the projectile nucleus (red) incident from the top, which follows a incoming Coulomb trajectory from infinity. The interaction with the target nucleus

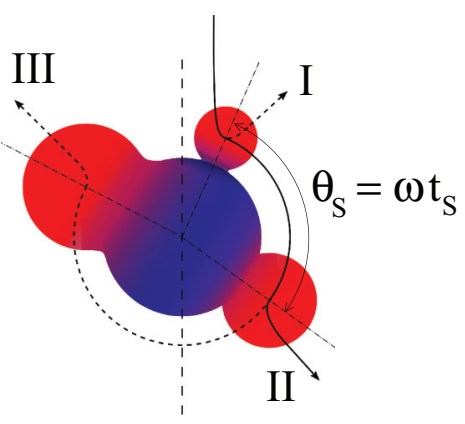

FIG. 14. (Color online) Schematic illustration of the basic physics of the Monte Carlo model used to simulate quasifission. Three different quasifission outcomes (I to III), depending on sticking time $\left(t_{s}\right)$ and rotation speed $(\omega)$, are indicated. (blue) starts as the two nuclei touch, and mass can be transferred between them. While the dinuclear system sticks together, for a time $t_{s}$, it rotates around its center of mass with an angular velocity $\omega$, assumed to be constant. Once the system separates the fragments again move on Coulomb trajectories towards infinity. The figure illustrates three outcomes with increasing sticking time (I, II, and III) during the first revolution of the system. To calculate the MAD according to the above picture the simulation model is divided into two parts, dealing with calculation of the observation angle of the fragments, and with the evolution of their masses during the sticking time.

The observed scattering angle, which depends on the sticking time $t_{s}$, is determined from

$$
\theta\left(t_{s}\right)=\pi-\Theta_{\text {in }}-\Theta_{s}\left(t_{s}\right)-\Theta_{\text {out }} .
$$

Here $\Theta_{s}\left(t_{s}\right)$ is the rotation angle of the system between contact and scission, and $\Theta_{\text {in }}$ and $\Theta_{\text {out }}$ are the relevant angles derived from classical Coulomb trajectories for the incoming and outgoing nuclei, respectively. $\Theta_{\text {in }}$ is the angle between the vector joining the centers of the two colliding nuclei at infinity (before the collision) and that at the distance of closest approach. Thus the relationship between the $\Theta_{\text {in }}$ and the Coulomb scattering angle $\theta_{\text {in }}$ with respect to the beam direction is $2 \Theta_{\text {in }}=\pi-\theta_{\text {in }} \cdot \theta_{\text {in }}$ is calculated in the usual way from the expression $\tan \left(\theta_{\text {in }} / 2\right)=D_{\text {in }} / 2 b_{\text {in }}$, where $b_{\text {in }}$ is the impact parameter associated with a given incident angular momentum. Thus $\Theta_{\text {in }}$ is calculated according to

$$
\Theta_{\text {in }}=\frac{\pi}{2}-\tan ^{-1} \frac{D_{\text {in }}}{2 b_{\text {in }}} .
$$

Evaluation of the equivalent angle $\Theta_{\text {out }}$ for the outgoing channel is slightly more complicated, involving estimation of the parameters $D_{\text {out }}$ and $b_{\text {out }}$. To determine $D_{\text {out }}$ we assume no radial velocity at scission, and that the fragment kinetic energies are fully damped, and follow Viola's total kinetic energy [53] systematics, accounting for asymmetric mass splits [54]. To evaluate $b_{\text {out }}$ we assume that $2 / 7$ of the initial angular momentum $J \hbar$ is converted to intrinsic angular momentum in the two fragments.

It is assumed that the two colliding nuclei touch and stick at the distance of closest approach $D_{\text {in }}$, evaluated for the incoming Coulomb trajectory. This is reasonable for the energies of interest near the capture barrier. The rotation angle during time $t_{s}$ between contact and scission is obtained from

$$
\theta_{s}\left(t_{s}\right)=\frac{\sqrt{J(J+1)} \hbar}{\langle I\rangle} t_{s} .
$$

Angular momenta are chosen randomly, weighted by the angular momentum distributions calculated using the couplechannels code CCFULL. This is the angular momentum distribution corresponding to capture in the entrance-channel potential pocket. As such it implicitly includes fusion-fission, quasifission, and any deep inelastic reaction processes that result after capture. The average moment of inertia $\langle I\rangle$ is estimated as the average of the entry $I_{\text {in }}$ and exit $I_{\text {out }}$ moments of inertia. In the similar picture of Tōke et al. [2], it was assumed the two nuclei were touching spheres at scission. For consistency with the mass equilibration time constant extracted 
using that model, it was decided to calculate both $I_{\text {in }}$ and $I_{\text {out }}$ using the distance between the two nuclei assuming they were touching spheres. In future, it should be possible to use dynamical models of quasifission to obtain better estimates of the mean moment of inertia, or even include a time-dependent moment of inertia.

The mass flow between the two constituents was modeled following Ref. [2], using an exponential function through which mass symmetry is reached asymptotically with time. This is the form expected [2] for overdamped motion in a parabolic potential with a minimum at $M_{R}=0.5$. The initial mass ratio $M_{R}(0)$ before mass exchange starts is the ratio of target mass to the sum of the projectile and target masses. The mass ratio evolution towards mass symmetry $\left(M_{R}=0.5\right)$ is calculated through

$$
M_{R}(t)=\left[M_{R}(0)-0.5\right] e^{-t_{s} / \tau_{m}}+0.5,
$$

where $\tau_{m}=5.2 \times 10^{-21} \mathrm{~s}$ is the mass equilibrium time constant determined by Tōke et al. [2], and $t_{s}$ is the sticking time as described above. For each event in the simulation the calculated final mass ratio associated with the randomly chosen sticking time was itself randomized around its average value to reflect the effects of fluctuations. The randomization was done using a Gaussian form, where the width varied linearly from $\sigma_{M_{R}}=0.025$ at the initial mass split to $\sigma_{M_{R}}=0.070$ at mass symmetry. These widths are compatible with experimental measurements of deep-inelastic and fusion-fission data, respectively $[8,13,50]$.

The distribution of sticking times was chosen to be a half-Gaussian rise followed by a matched exponential fall. The width of the half-Gaussian (rise time) and the fall time were variables adjusted to reproduce measured MAD. Different combinations of rise and fall times gave similar outcomes, but the mean time was quite well defined by the experimental MAD [34]. Later work will make a detailed quantitative comparison of measured and calculated MAD, to determine how much information on quasifission timescales can be reliably extracted from MAD measurements.

\section{APPENDIX C: FISSILITY PARAMETERS}

The experimental MAD have been ordered in terms of two fissility parameters. The compound nucleus fissility parameter $x_{\mathrm{CN}}$ is a scaling parameter reflecting the ratio of the competing Coulomb and nuclear forces for compact shapes. Following the formalism of Ref. [41], it is given by

$$
x_{\mathrm{CN}}=\frac{\left(Z^{2} / A\right)}{\left(Z^{2} / A\right)_{\mathrm{crit}}},
$$

where $Z$ and $A$ is the proton and mass numbers of the compound nucleus, respectively. The $\left(Z^{2} / A\right)_{\text {crit }}$ is taken as

$$
\left(Z^{2} / A\right)_{\text {crit }}=50.883\left(1-1.7826 I^{2}\right),
$$

where $I=(A-2 Z) / A$ is the relative neutron excess of the compound nucleus in question.

The second fissility parameter used is the effective entrance channel fissility parameter $x_{\text {eff }}$. This includes the effect of mass and charge asymmetry [40]. It was used extensively $[35,41,55,56]$ by itself, or in combination with $x_{\mathrm{CN}}$, to determine a single scaling parameter which would be able to predict the behavior of any system. It is given by

$$
x_{\text {eff }}=\frac{4 Z_{1} Z_{2} /\left[A_{1}^{1 / 3} A_{2}^{1 / 3}\left(A_{1}^{1 / 3}+A_{2}^{1 / 3}\right)\right]}{\left(Z^{2} / A\right)_{\text {crit }}} .
$$

Using the above equations $x_{\mathrm{CN}}$ and $x_{\text {eff }}$ have been calculated for each target-projectile combination investigated. The resulting values are shown in Table I.

A comparison between the characteristics of the experimental MAD and the effective fissility $x_{\text {eff }}$ was made by including curves of constant fissility in Fig. 2 (solid lines with values of fissility marked). The fissility lines were generated by first calculating the effective fissility for each even-even projectile-target combination using $2 \leqslant Z_{p} \leqslant 40$ and $16 \leqslant Z_{t} \leqslant 114$, where $Z_{p}$ and $Z_{t}$ is the number of protons for projectile and target nucleus, respectively. The isotopes used in the calculations, and hence the mass numbers, were in each case chosen to lie along the valley of beta stability, as approximated by Green's formula

$$
Z / A=0.5[1-0.4 A /(200+A)] .
$$

The atomic number and mass number for the corresponding compound nucleus was calculated as $Z_{\mathrm{CN}}=Z_{p}+Z_{t}$ and $A_{\mathrm{CN}}=A_{p}+A_{t}$, respectively.

Following Blocki [41], a mean fissility $\left(x_{m}\right)$ can be parametrized as a linear combination of the compound nucleus fissility, $x_{\mathrm{CN}}$, and the effective entrance channel fissility $x_{\mathrm{eff}}$ for the target-projectile combination, such that $x_{m}=g x_{\mathrm{CN}}+$ $(1-g) x_{\text {eff }}$, where $g$ is the weighting factor. The factor $g$ was determined empirically in this work from the systematic changes in MAD characteristics.
[1] B. B. Back, Phys. Rev. C 31, 2104 (1985).

[2] J. Tōke, B. Bock, G. X. Dai, A. Gobbi, S. Gralla, K. D. Hildenbrand, J. Kuzminski, W. Müller, A. Olmi, and H. Stelzer, Nucl. Phys. A 440, 327 (1985).

[3] W. Q. Shen, J. Albinski, A. Gobbi, S. Gralla, K. D. Hildenbrand, N. Herrmann, J. Kuzminski, W. F. J. Müller, H. Stelzer, J. Tōke, B. B. Back, S. Bjørnholm, and S. P. Sørensen, Phys. Rev. C 36, 115 (1987).
[4] C. C. Sahm, H. G. Clerc, K.-H. Schmidt, W. Reisdorf, P. Armbruster, F. P. Hessberger, J. G. Keller, G. Munzenberg, and D. Vermeulen, Z. Phys. A 319, 113 (1984).

[5] A. C. Berriman, D. J. Hinde, M. Dasgupta, C. R. Morton, R. D. Butt, and J. O. Newton, Nature (London) 413, 144 (2001).

[6] D. J. Hinde, M. Dasgupta, and A. Mukherjee, Phys. Rev. Lett. 89, 282701 (2002). 
[7] D. J. Hinde and M. Dasgupta, Phys. Lett. B 622, 23 (2005).

[8] D. J. Hinde, M. Dasgupta, J. R. Leigh, J. P. Lestone, J. C. Mein, C. R. Morton, J. O. Newton, and H. Timmers, Phys. Rev. Lett. 74, 1295 (1995).

[9] D. J. Hinde, M. Dasgupta, J. R. Leigh, J. C. Mein, C. R. Morton, J. O. Newton, and H. Timmers, Phys. Rev. C 53, 1290 (1996).

[10] G. N. Knyazheva, E. M. Kozulin, R. N. Sagaidak, A. Y. Chizhov, M. G. Itkis, N. A. Kondratiev, V. M. Voskressensky, A. M. Stefanini, B. R. Behera, L. Corradi, E. Fioretto, A. Gadea, A. Latina, S. Szilner, M. Trotta, S. Beghini, G. Montagnoli, F. Scarlassara, F. Haas, N. Rowley, P. R. S. Gomes, and A. S. d. Toledo, Phys. Rev. C 75, 064602 (2007).

[11] K. Nishio, H. Ikezoe, S. Mitsuoka, I. Nishinaka, Y. Nagame, Y. Watanabe, T. Ohtsuki, K. Hirose, and S. Hofmann, Phys. Rev. C 77, 064607 (2008).

[12] K. Nishio, S. Mitsuoka, I. Nishinaka, H. Makii, Y. Wakabayashi, H. Ikezoe, K. Hirose, T. Ohtsuki, Y. Aritomo, and S. Hofmann, Phys. Rev. C 86, 034608 (2012).

[13] C. Simenel, D. J. Hinde, R. du Rietz, M. Dasgupta, M. Evers, C. J. Lin, D. H. Luong, and A. Wakhle, Phys. Lett. B 710, 607 (2012).

[14] T. Cap, K. Siwek-Wilczyńska, and J. Wilczyński, Phys. Rev. C 83, 054602 (2011).

[15] K. Siwek-Wilczyńska, T. Cap, M. Kowal, A. Sobiczewski, and J. Wilczyński, Phys. Rev. C 86, 014611 (2012).

[16] V. I. Zagrebaev, A. V. Karpov, and W. Greiner, Phys. Rev. C 85, 014608 (2012).

[17] W. Loveland, J. Phys. Conf. Ser. 420, 012004 (2013).

[18] Y. Aritomo, Phys. Rev. C 75, 024602 (2007).

[19] V. I. Zagrebaev and W. Greiner, J. Phys. G: Nucl. Part. Phys. 31, 825 (2005).

[20] V. Zagrebaev, A. Karpov, M. Aritomo, Y. Naumenko, and W. Greiner, Phys. Part. and Nucl. 38, 469 (2007).

[21] W. J. Swiatecki, K. Siwek-Wilczyńska, and J. Wilczyński, Phys. Rev. C 71, 014602 (2005).

[22] G. Adamian, N. Antonenko, W. Scheid, and V. Volkov, Nucl. Phys. A 633, 409 (1998).

[23] Y. Aritomo, K. Hagino, K. Nishio, and S. Chiba, Phys. Rev. C 85, 044614 (2012).

[24] R. Bock, Y. T. Chu, M. Dakowski, A. Gobbi, E. Grosse, A. Olmi, H. Sann, D. Schwalm, U. Lynen, W. Muller, S. Bjornholm, H. Esbensen, W. Wolfli, and E. Morenzoni, Nucl. Phys. A 388, 334 (1982).

[25] D. J. Hinde, R. G. Thomas, R. du Rietz, A. Diaz-Torres, M. Dasgupta, M. L. Brown, M. Evers, L. R. Gasques, R. Rafiei, and M. D. Rodriguez, Phys. Rev. Lett. 100, 202701 (2008).

[26] D. J. Hinde, R. du Rietz, M. Dasgupta, R. G. Thomas, and L. R. Gasques, Phys. Rev. Lett. 101, 092701 (2008).

[27] E. V. Prokhorova, A. A. Bogachev, M. G. Itkis, I. M. Itkis, G. Knyazheva, N. A. Kondratiev, E. M. Kozulin, L. Krupa, Y. T. Oganessian, I. V. Pokrovsky, V. V. Pashkevich, and A. Y. Rusanov, Nucl. Phys. A 802, 45 (2008).

[28] I. M. Itkis, E. M. Kozulin, M. G. Itkis, G. N. Knyazheva, A. A. Bogachev, E. V. Chernysheva, L. Krupa, Y. T. Oganessian, V. I. Zagrebaev, A. Y. Rusanov, F. Goennenwein, O. Dorvaux, L. Stuttgé, F. Hanappe, E. Vardaci, and E. de Goés Brennand, Phys. Rev. C 83, 064613 (2011).
[29] R. G. Thomas, D. J. Hinde, D. Duniec, F. Zenke, M. Dasgupta, M. L. Brown, M. Evers, L. R. Gasques, M. D. Rodriguez, and A. Diaz-Torres, Phys. Rev. C 77, 034610 (2008).

[30] W. J. Swiatecki, K. Siwek-Wilczyńska, and J. Wilczyński, Int. J. Mod. Phys. E 13, 261 (2004).

[31] R. Rafiei, R. G. Thomas, D. J. Hinde, M. Dasgupta, C. R. Morton, L. R. Gasques, M. L. Brown, and M. D. Rodriguez, Phys. Rev. C 77, 024606 (2008).

[32] C. J. Lin, R. du Rietz, D. J. Hinde, M. Dasgupta, R. G. Thomas, M. L. Brown, M. Evers, L. R. Gasques, and M. D. Rodriguez, Phys. Rev. C 85, 014611 (2012).

[33] E. Williams, D. J. Hinde, M. Dasgupta, R. du Rietz, I. P. Carter, M. Evers, D. H. Luong, S. D. McNeil, D. C. Rafferty, K. Ramachandran, and A. Wakhle, Phys. Rev. C 88, 034611 (2013).

[34] R. du Rietz, D. J. Hinde, M. Dasgupta, R. G. Thomas, L. R. Gasques, M. Evers, N. Lobanov, and A. Wakhle, Phys. Rev. Lett. 106, 052701 (2011).

[35] S. Bjornholm and W. Swiatecki, Nucl. Phys. A 391, 471 (1982).

[36] K. E. Rehm, A. M. van den Berg, J. J. Kolata, D. G. Kovar, W. Kutschera, G. Rosner, G. S. F. Stephans, and J. L. Yntema, Phys. Rev. C 37, 2629 (1988).

[37] W. Reisdorf, J. Phys. G 20, 1297 (1994).

[38] D. J. Hinde, D. Hilscher, H. Rossner, B. Gebauer, M. Lehmann, and M. Wilpert, Phys. Rev. C 45, 1229 (1992)

[39] K. Hagino, N. Rowley, and A. T. Kruppa, Comput. Phys. Commun. 129, 143 (1999).

[40] R. Bass, Nucl. Phys. A 231, 45 (1974).

[41] J. Blocki, H. Feldmeier, and W. Swiatecki, Nucl. Phys. A 459, 145 (1986).

[42] A. Wakhle et al. (unpublished).

[43] J. C. Mein, D. J. Hinde, M. Dasgupta, J. R. Leigh, J. O. Newton, and H. Timmers, Phys. Rev. C 55, R995 (1997).

[44] S. Mitsuoka, H. Ikezoe, K. Nishio, K. Satou, and J. Lu, Phys. Rev. C 65, 054608 (2002).

[45] A. Y. Chizhov, M. G. Itkis, I. M. Itkis, G. N. Kniajeva, E. M. Kozulin, N. A. Kondratiev, I. V. Pokrovsky, R. N. Sagaidak, V. M. Voskressensky, A. V. Yeremin, L. Corradi, A. Gadea, A. Latina, A. M. Stefanini, S. Szilner, M. Trotta, A. M. Vinodkumar, S. Beghini, G. Montagnoli, F. Scarlassara, A. Y. Rusanov, F. Hanappe, O. Dorvaux, N. Rowley, and L. Stuttgé, Phys. Rev. C 67, 011603 (2003).

[46] M. G. Itkis, J. Äystö, S. Beghini, A. A. Bogachev, L. Corradi, O. Dorvaux, A. Gadea, G. Giardina, F. Hanappe, I. M. Itkis, M. Jandel, J. Kliman, S. V. Khlebnikov, G. N. Kniajeva, N. A. Kondratiev, E. M. Kozulin, L. Krupa, A. Latina, T. Materna, G. Montagnoli, Y. T. Oganessian, I. V. Pokrovsky, E. V. Prokhorova, N. Rowley, V. A. Rubchenya, A. Rusanov, R. N. Sagaidak, F. Scarlassara, A. M. Stefanini, L. Stuttge, S. Szilner, M. Trotta, W. H. Trzaska, D. N. Vakhtin, A. M. Vinodkumar, V. M. Voskressenski, and V. I. Zagrebaev, Nucl. Phys. A 734, 136 (2004).

[47] R. Kalpakchieva, Y. Oganessian, Y. Penionzhkevich, and H. Sodan, Z. Phys. A 283, 253 (1977).

[48] M. Morjean, D. Jacquet, J. L. Charvet, A. L'Hoir, M. Laget, M. Parlog, A. Chbihi, M. Chevallier, C. Cohen, D. Dauvergne, R. Dayras, A. Drouart, C. Escano-Rodriguez, J. D. Frankland, R. Kirsch, P. Lautesse, L. Nalpas, C. Ray, C. Schmitt, C. Stodel, 
L. Tassan-Got, E. Testa, and C. Volant, Phys. Rev. Lett. 101, 072701 (2008).

[49] Y. Aritomo, Phys. Rev. C 80, 064604 (2009).

[50] G. Guarino, A. Gobbi, K. D. Hildenbrand, W. F. J. Müller, A. Olmi, H. Sann, S. Bjørnholm, and G. Rudolf, Nucl. Phys. A 424, 157 (1984).

[51] D. J. Hinde, R. du Rietz, M. Dasgupta, R. G. Thomas, and L. R. Gasques, Phys. Rev. Lett. 101, 092701 (2008).
[52] D. J. Hinde, R. L. Ahlefeldt, R. G. Thomas, K. Hagino, M. L. Brown, M. Dasgupta, M. Evers, L. R. Gasques, and M. D. Rodriguez, Phys. Rev. C 76, 014617 (2007).

[53] V. E. Viola, K. Kwiatkowski, and M. Walker, Phys. Rev. C 31, 1550 (1985).

[54] D. J. Hinde, J. R. Leigh, J. J. M. Bokhorst, J. O. Newton, R. L. Walsh, and J. W. Boldeman, Nucl. Phys. A 472, 318 (1987).

[55] W. J. Swiatecki, Phys. Scr. 24, 113 (1981).

[56] W. J. Swiatecki, Nucl. Phys. A 376, 275 (1982). 